\title{
LO STATO SOCIALE NELL'ERA \\ DELL'AUSTERITÀ PERMANENTE
}

\author{
di Paul Pierson
}

Da tempo riconosciuto come un elemento caratterizzante delle democrazie industriali avanzate, il welfare state reclama oggi più attenzione che mai. Malgrado l'incremento delle disuguaglianze in molti paesi, le domande di tagli alla spesa sociale si sono intensificate. I tentativi in tale senso hanno provocato un acceso dibattito e diffuse proteste e ciò pone il welfare al centro del dibattito politico e del conflitto sociale.

Gli osservatori spesso dipingono tali conflitti come sintomi di profonde divergenze tra sostenitori e detrattori dei principi basilari del contratto sociale post-bellico. Essi hanno spesso sottolineato che di fronte alle trasformazioni dell'economia globale, le cui conseguenze hanno inferto un duro colpo ai fondamenti sociali ed economici del welfare, i margini di manovra politica per i provvedimenti di natura redistributiva sono ormai inesistenti. Pur contenendo alcuni elementi di verità, tali descrizioni non colgono però le caratteristiche di fondo delle attuali politiche di welfare. Se i mutamenti nell'economia globale sono importanti, le pressioni sui sistemi di welfare maturi sono infatti imputabili anzitutto alle trasformazioni socio-economiche in atto nelle democrazie del benessere, all'interno delle quali, quasi ovunque, il sostegno nei confronti del welfare state appare ancora esteso.

In questo lavoro si sostiene che le odierne politiche di welfare sono plasmate tanto da una spinta verso l'austerità quanto $\mathrm{da}$ un consenso di fondo che permane inalterato nel tempo. Nella maggior parte delle democrazie del benessere la politica delle politiche sociali è incentrata più sulla rinegoziazione, ristrutturazione e modernizzazione del contratto sociale post-bellico che non sul suo smantellamento. La questione cruciale è comprendere se particolari contesti nazionali facilitino l'emerge- 
re di un siffatto sforzo di riforma - una riforma «centrista» - e quali, eventualmente, ne siano i termini.

Il saggio si articola in tre passaggi. Nel primo si tratteggia uno schema di riferimento per lo studio della politica di rifor$\mathrm{ma}$ in un contesto di austerità permanente. Con il secondo si introducono due fattori che rendono l'analisi più complessa: l'opportunità di considerare più dimensioni della riforma della politica sociale e la necessità di riconoscere, nel novero delle democrazie del benessere, tre diverse configurazioni di politiche di welfare. Tali argomentazioni, infine, vengono impiegate per analizzare la politica della ristrutturazione del welfare state nei tre diversi «mondi» di capitalismo di welfare: liberale, socialdemocratico e conservatore.

La politica dell'austerità permanente. Uno schema preliminare

Pur nel dissenso circa le cause del fenomeno, l'idea che l'odierno stato sociale debba fronteggiare un contesto di austerità permanente è condivisa da tutti gli osservatori. Infatti, i mutamenti nell'economia globale, il consistente rallentamento della crescita economica, la maturazione degli impegni di governo e l'invecchiamento della popolazione sono alla radice di un significativo stress fiscale.

Evidenziare le forti pressioni a cui i sistemi di welfare maturi sono sottoposti non implica, tuttavia, che il risultato atteso sia il collasso o il loro radicale ridimensionamento. Come ho evidenziato altrove (Pierson 1994; 1996), la forza politica dei sistemi di welfare dipende da cause diverse, ascrivibili a due categorie principali: gli incentivi elettorali connessi con programmi di welfare che conseguono un forte sostegno nella popolazione; e la «vischiosità» istituzionale, che limita fortemente le possibilità di riforma.

Incentivi elettorali. Nelle democrazie di mercato gli elettori ricoprono un ruolo cruciale. L'attuazione di riforme politiche non può prescindere di solito da una sanzione elettorale. La crescita del welfare ha determinato l'espansione delle sue costituencies: come ha osservato Peter Flora, «includendo i beneficiari delle prestazioni (pensionistiche) contro la disoccupazione e di assistenza sociale nonché il personale impiegato nel settore educativo, nel settore sanitario e nei servizi sociali, oggi in molti 
paesi un elettore su due riceve trasferimenti monetari o stipendi grazie al welfare state» $(1989,154)$.

Per molti aspetti tali stime sottovalutano in misura considerevole il peso del welfare nella vita politica contemporanea. Molti degli elettori che non ricevono prestazioni di welfare probabilmente se le aspettano in futuro; altri vivono con familiari che beneficiano di prestazioni di welfare o che hanno un impiego ad esso connesso. Nella maggior parte dei paesi i programmi universalistici, specialmente ma non esclusivamente in campo sanitario, godono di un ampio sostegno; inoltre, il welfare mantiene una legittimità considerevole come fonte di stabilità e garante dei principi fondamentali di cittadinanza. Pertanto quasi ovunque il sostegno della popolazione sembra estendersi ben oltre i confini del ristretto self-interest economico.

Generalmente, il sostegno a favore del welfare non è soltanto ampio, ma anche intenso. L'intensità della preferenza è rilevante perché è connessa con tassi più alti di mobilitazione politica e con le reali scelte partitiche degli elettori. Tale intensità dipende da due fattori. In primo luogo, mentre i benefici del ridimensionamento del welfare sono generalmente diffusi e spesso incerti, la maggior parte delle constituencies interessate dalle prestazioni detiene un interesse «concentrato» nei confronti del loro mantenimento. E di solito, secondo uno dei pochi assiomi della scienza politica, gli interessi concentrati prevalgono su quelli diffusi.

I sostenitori del welfare, in secondo luogo, difendono benefici già esistenti, e ciò rafforza l'intensità delle loro preferenze. Gli studiosi di comportamento elettorale e di psicologia politica ritengono che gli elettori siano dotati di un bias negativo: reagiscono più intensamente alle perdite che ai commisurati vantaggi potenziali. Pertanto, la base elettorale del welfare state non soltanto è molto ampia, ma è anche determinata a punire i politici responsabili di iniziative impopolari.

E necessario inoltre far cenno anche a un altro aspetto della dimensione elettorale: il limitato orizzonte temporale di riferimento. Poiché, di norma, i politici desiderano essere rieletti, è possibile che non considerino affatto gli effetti di lungo periodo di un'eventuale riforma. Il ridimensionamento dello stato sociale può offrire benefici soltanto in un tempo successivo all'immediato manifestarsi dei suoi effetti negativi. L'asimmetria connessa alla presenza di costi di breve termine e benefici di lungo periodo disincentiva ulteriormente $\mathrm{i}$ politici dall'intraprendere 
iniziative volte ad una radicale riduzione delle prestazioni di welfare.

Vischiosità istituzionale. I soggetti che cercano di esercitare pressioni per una riforma di welfare devono confrontarsi non soltanto con l'opposizione degli elettori e dei beneficiari dei programmi di welfare, ma anche con la vischiosità degli assetti di policy che informano le politiche sociali. In particolare, vi sono due aspetti dei sistemi politici sviluppati che rafforzano gli ostacoli di natura elettorale frapposti ad una riforma radicale: $i$ «punti di veto» (veto points) formali e informali e i processi di path dependency, che spesso causano il congelamento degli assetti di policy esistenti. Entrambi tendono a indirizzare i progetti di riforma nella direzione di aggiustamenti incrementali.

Le peculiarità dei veto points sono espresse chiaramente da Bonoli (in stampa) ${ }^{1}$. La maggior parte dei sistemi politici include assetti istituzionali che richiedono più di una maggioranza semplice per l'adozione di una riforma, offrendo in tal modo la possibilità a minoranze (talvolta minoranze molto piccole) di impedire l'azione di riforma. Esempi di tali assetti sono un sistema federale, un forte potere giudiziario e/o il bicameralismo, la possibilità di indire referendum, maggioranze qualificate e governi di coalizione ${ }^{2}$. Non solo è estremamente difficile creare una coalizione di maggioranza favorevole alla ristrutturazione dello stato sociale, ma talvolta può anche non essere sufficiente. La moltiplicazione dei veto points può interrompere qualsiasi tentativo di policy change, come è accaduto ad esempio per una ambiziosa e aggressiva coalizione di riforma come la maggioranza repubblicana nel Congresso statunitense post-1994 o per la coalizione conservatrice tedesca al governo dal 1982 al 1998.

La seconda fonte di vischiosità istituzionale è più complessa. Poiché il concetto di path dependence è spesso utilizzato come fattore esplicativo senza motivazione esplicita, è necessario chiarire la nostra scelta ${ }^{3}$. Alcuni percorsi di sviluppo politico, una volta avviati, risultano difficili da modificare. Tale diffi-

1 Si vedano, più in generale, anche Scharpf (1986) e Tsebelis (1995).

2 Il veto può anche essere riferito a singoli aspetti di policy. Il sistema previdenziale canadese, ad esempio, non può essere riformato senza che vi sia una maggioranza qualificata dei governi provinciali.

3 Un'analisi più circostanziata si trova in Pierson (1997). Si vedano anche Myles (in stampa) e Pierson e Wood (in stampa). 
coltà non è causata soltanto dalla presenza di veto points istituzionali. Gli adattamenti individuali e organizzativi ad assetti precedenti possono contribuire a rendere il mutamento poco appetibile. Quando gli attori si adattano alle nuove regole del gioco, è possibile che le azioni intraprese in passato blocchino opzioni altrimenti accessibili. In altre parole, l'adattamento sociale agli assetti istituzionali e organizzativi incrementa notevolmente i costi di exit dall'assetto esistente. Pertanto, alternative disponibili in passato possono risultare inaccessibili nel presente. Tutto ciò determina, come è stato enfatizzato da Douglass North, la natura incrementale dei mutamenti nei sistemi politici solidamente istituzionalizzati.

Le ricerche sui mutamenti tecnologici hanno illustrato alcune delle circostanze che conducono alla path dependence (David 1985; Arthur 1994). Come è stato argomentato da North, i risultati di tali ricerche possono essere estesi dal mutamento tecnologico ad altri mutamenti sociali, in particolare allo sviluppo delle istituzioni (North 1990). In un contesto di elevata complessità sociale, la costruzione di nuove istituzioni implica spesso alti costi fissi o iniziali, può dare luogo a notevoli «effetti di apprendimento» e generare «effetti di coordinamento» e «aspettative di adattamento». Istituzioni consolidate generano consistenti incentivi che rafforzano la loro stabilità e il loro sviluppo futuro.

Ciò naturalmente non significa che la path dependence «congeli» gli assetti esistenti. La politica di welfare e i sistemi di welfare contemporanei sono caratterizzati da numerosi effetti di path dependency. I sistemi previdenziali ne sono un chiaro esempio. La maggior parte dei paesi opera con un sistema a ripartizione: se utilizzati per un lungo periodo di tempo, i sistemi a ripartizione possono subire limature e aggiustamenti, ma sono molto resistenti ad una riforma radicale. Passare ad un sistema privato determinerebbe un peso eccessivo per gli attuali lavoratori, che si troverebbero a dover finanziare le pensioni della generazione precedente e contestualmente accumulare risorse sufficienti per le proprie.

Analoghi effetti di path dependency, anche se meno consistenti, possono realizzarsi in settori di politica sociale in cui sistemi complessi di istituzioni e organizzazioni hanno conosciuto una evoluzione simile nel corso del tempo (Giaimo e Manow 1999; Hacker 1998). Nel settore sanitario, e per molti aspetti nei mercati del lavoro, gli attori sociali devono coordinare le 
loro attività e investire risorse sulla base delle strutture degli incentivi esistenti. Tali aspetti contribuiscono a spiegare, ad esempio, perché i datori di lavoro si sono mostrati spesso più esitanti e internamente divisi nei confronti di una riforma di policy, rispetto a quanto previsto dalle teorie di politica economica (Thelen in stampa).

La popolarità dello stato sociale e la prevalenza di caratteri di vischiosità devono entrambe collocarsi al centro delle analisi sulla ristrutturazione dei sistemi di welfare. Per gli studiosi di politiche pubbliche l'interrogativo principale si pone in questi termini: cosa succede quando le forze inarrestabili della post-industrializzazione si trovano davanti l'immutabilità dello stato sociale? Conoscere la forza di entrambi i soggetti dello scontro determina molte conseguenze significative nell'analisi delle politiche di riforma.

In molti paesi vi sono solide ragioni che giustificano un atteggiamento diffidente nei confronti della possibilità di una riforma radicale dei sistemi di welfare. In pochissimi casi i politici sono stati in grado di dar vita e sostenere una coalizione di maggioranza, riuscendo ad ottenere una consistente contrazione della politica sociale (Stephens, Huber e Ray 1999). Invece, in molti paesi l'ampio sostegno pubblico, l'intensità delle preferenze espresse dai beneficiari delle prestazioni di welfare, l'estensione della capacità di adattamento da parte di una molteplicità di attori (inclusi i datori di lavoro) nei confronti dell'economia sociale di mercato e gli assetti istituzionali favorevoli alla difesa dello status quo rendono un «assalto» allo stato sociale politicamente suicida.

Ciononostante, le pressioni connesse alla post-industrializzazione, rafforzate per certi versi dalla globalizzazione, hanno reso il mantenimento dello status quo un'opzione progressivamente irrealistica. La persistenza di una crescita economica limitata, unitamente alla difficoltà di creare opportunità di lavoro nel settore dei servizi, all'invecchiamento della popolazione e alla difficoltà di far fronte alle garanzie offerte dalle politiche esistenti, genera forti pressioni verso il mutamento. Inoltre, non solo i livelli di imposizione fiscale stanno superando la soglia di tolleranza massima della popolazione, ma anche il peso dei contributi sociali sul costo del lavoro e il loro possibile impatto negativo sull'occupazione e sui salari creano forti tensioni all'interno delle tradizionali coalizioni favorevoli allo stato sociale, in particolare tra le rappresentanze sindacali del settore privato 
(Visser e Hemerijck 1997). Poiché il ritorno a una fase di alta crescita economica appare fortemente improbabile, le pressioni fiscali sullo stato sociale sono destinate ad intensificarsi. Mentre gli incrementi nell'imposizione fiscale possono contribuire all'eliminazione del divario esistente tra le prestazioni offerte e le risorse disponibili, risulta difficile pensare che in molti paesi europei semplici mutamenti nei livelli salariali possano essere sufficienti al mantenimento dell'equilibrio fiscale. Pertanto, anche convinti sostenitori dello stato sociale sono sempre più consapevoli del fatto che lasciare immutato l'impianto originario richiede riforme significative.

In tale contesto, è probabile che i conflitti intorno alla ristrutturazione delle politiche sociali si trasformino. Nei paesi in cui aggressivi propugnatori del neoliberismo si sono trovati al potere, come ad esempio in Nuova Zelanda e fino a tempi recenti nel Regno Unito, lo scontro è avvenuto prevalentemente tra sostenitori dello status quo e soggetti desiderosi di smantellare lo stato sociale. In altri casi, in un clima in cui le tendenze sociali hanno reso consistenti ed inevitabili le pressioni sui bilanci, è probabile che i cleavages politici diventino più complessi. I sostenitori di una ristrutturazione annoverano tra le proprie fila molti soggetti che desiderano mantenere e modernizzare aspetti essenziali del contratto sociale, ma al contempo cercano di ottenere tale risultato in modo da non creare carichi fiscali insostenibili, da contribuire alla crescita economica e da consentire a nuove domande sociali di entrare in competizione con domande sociali consolidate. È pertanto necessario distinguere tra ristrutturazione, da un lato, e riduzione o smantellamento, dall'altro.

Fin qui ho sostenuto la tesi dell'improbabile praticabilità sia dell'immobilismo, sia dello smantellamento, nella maggior parte dei paesi. Come in molte altre dimensioni della politica, dovremmo invece aspettarci l'emergere di forti spinte verso risposte più centriste - quindi più incrementali. Il potere politico sarà in genere nelle mani di attori che puntano a una significativa riduzione dei costi e, contestualmente, a una modernizzazione di alcuni aspetti delle prestazioni sociali.

Un proficuo schema iniziale per motivare tale affermazione è rappresentato da una versione semplificata della tesi di pivotal politics suggerita da alcuni recenti studi sulla politica statunitense (Krehbiel 1998; Brady e Volden 1998). In una qualsiasi circostanza di scelta collettiva in cui le preferenze di policy posso- 
no essere disposte su un unico continuum, vi è un attore centrale (pivotal) il cui voto risulta determinante perché un'iniziativa abbia seguito o viceversa venga bloccata. Gli elettori centrali non devono necessariamente essere elettori mediani: molto dipende dall'ambiente istituzionale in cui le preferenze maturano. A seconda della consistenza dei veto points e della presenza o meno di sistemi fortemente maggioritari, l'elettore centrale è più vicino allo status quo rispetto all'elettore mediano - spesso, molto più vicino. Ad esempio, negli Stati Uniti, se un Presidente è deciso a porre il veto su di una legge, l'attore centrale nell'organo legislativo non sarà l'attore che determina la maggioranza semplice, bensì quello che contribuirà a costituire una maggioranza qualificata dei due terzi, necessaria per superare il veto presidenziale.

In pratica, la vulnerabilità politica di chi cerca di modificare programmi di welfare comunemente apprezzati è tale da richiedere un consenso relativamente ampio per la riforma, e non una «coalizione minima-vincente». Un consenso più ampio legittima la richiesta di un mutamento di policy inteso a sostenere piuttosto che a distruggere il programma in esame (Weaver 1986). La volontà di rendere duratura una riforma spinge di solito nella stessa direzione. Più in particolare, con programmi ampi, complessi e profondamente istituzionalizzati come il sistema sanitario e pensionistico, è probabile che gli attori sociali considerino di grande importanza la prevedibilità e la continuità della politica in questione. Una semplice riforma non è sufficiente: forti interessi ricercano una ragionevole garanzia che le nuove politiche possano godere di un certo sostegno. Ciò incoraggia la formazione di ampie coalizioni, spesso informali e legate a una specifica issue, e quindi l'estensione del ventaglio di attori, riservando loro parte dei benefici conseguibili con la riforma e rafforzando la convinzione che la successiva elezione non determinerà uno stravolgimento delle nuove iniziative.

Un ultimo fattore che contribuisce ad indirizzare l'attore strategico verso lo status quo è la maggiore o minore credibilità dei governi che propongono la riforma (Ross 1998). Spesso sono i governi caratterizzati da un atteggiamento tradizionalmente favorevole allo stato sociale ad avere maggiori spazi di manovra. Tuttavia, gli stessi fattori che determinano tale credibilità (gli impegni assunti in passato, l'orientamento ideologico, la natura delle constituencies ad esso più vicine) rendono difficile l'eventualità che i partiti avvantaggiati da tali condizioni le useranno per smantellare politiche sociali consolidate. 
Gli studiosi di politiche pubbliche in chiave comparata hanno riconosciuto da tempo il ruolo di un gioco a somma positiva tra le più rilevanti organizzazioni di rappresentanza degli interessi e i partiti politici. In molte democrazie industriali avanzate il nuovo ambiente economico e fiscale è stato trasformato. Non altrettanto può dirsi delle condizioni necessarie per una politica basata sullo scambio e volta ad ottenere consenso. È cambiata la «valuta» necessaria per effettuare tali scambi. Tradizionalmente, il contributo del mondo del lavoro è rappresentato dalla moderazione salariale. I riformatori dello stato sociale, tuttavia, necessitano anche di credibilità e legittimità, in particolare di fronte agli elettori. Generalmente, partiti di sinistra e centro-sinistra, e/o associazioni sindacali, devono essere inseriti nella coalizione di riforma per rendere la ristrutturazione dei sistemi di welfare politicamente sostenibile. In alcuni casi (ad esempio, Germania, Italia e Paesi bassi) i sindacati sono stati partner attivi in tali accordi di riforma, in altri (è il caso della recente riforma pensionistica svedese) sono stati per lo più trascurati.

Le implicazioni di quanto detto finora sono evidenziate nella figura 1. Collocando le riforme su un continuum che va dallo status quo (q) a una riforma neoliberale estrema, di radicale smantellamento (s), è possibile attendersi che l'elettore mediano si collochi ad una considerevole distanza da (s). L'esigenza di superare i veto points spinge l'agenda di riforma del governo a tornare a $(\mathrm{v})$, e la volontà di ottenere un consenso che incrementi la legittimità e induca alla stabilità spinge ad un passo ulteriore verso (c). Pertanto, ritengo che nella maggior parte delle democrazie del benessere le riforme praticabili si collocheranno al massimo tra (c) e (v). La riforma, dunque, comporta un sostanziale allontanamento da (q), ma essa è anche distante da (s).

\begin{tabular}{llll}
\hline $\mathrm{Q}$ & $\mathrm{C}$ & $\mathrm{V}$ & $\mathrm{S}$
\end{tabular}

FIG. 1. Il posizionamento delle opzioni riformiste.

\section{Dimensioni di analisi e modelli di welfare state}

Fin qui ho tratteggiato un quadro estremamente semplificato delle politiche di riforma. Una descrizione più soddisfacente necessita di rendere l'analisi più complessa su due fronti crucia- 
li: sviluppare una più articolata concettualizzazione dell'agenda di riforma, ovvero, nel lessico proprio delle scienze sociali, della variabile dipendente; considerare le peculiarità che le dinamiche di riforma assumono nei diversi regimi di welfare state. L'una e l'altra di queste esigenze costituiscono una parte importante dell'attuale agenda di ricerca sulle politiche di welfare.

Le tre dimensioni della ristrutturazione dello stato sociale. Uno degli elementi caratteristici dell'attuale stato della ricerca sulle politiche di welfare è la mancanza di un consenso sugli esiti. Quanto, e in che modo, sono cambiati i sistemi di welfare dalla fine del boom postbellico? Alcuni studiosi ritengono che le riforme siano state incrementali e non radicali, incentrate su un processo di ristrutturazione e non di smantellamento (Pierson in stampa). Altri sostengono invece che le riduzioni sono state più consistenti (Clayton e Pontusson 1999). Tali problemi sono tuttavia almeno in parte superabili se si adotta una prospettiva analitica che pone al centro del processo di riforma gli attori, rendendo in tal modo possibile l'analisi delle tre dimensioni del mutamento: de-commodification o ri-mercificazione (ovvero il grado in cui il processo di riforma incoraggia il rientro nel mercato o il ricorso a mezzi propri), contenimento dei costi e modernizzazione. Ognuna costituisce una dimensione rilevante per la ristrutturazione dello stato sociale.

Mercificazione. Nell'incisiva formulazione di Esping-Andersen, la demercificazione «si realizza quando una prestazione diventa un diritto acquisito, e quando un individuo può ottenere $\mathrm{i}$ propri mezzi di sussistenza senza dipendere dal mercato» (1990, 21-22). Esping-Andersen considerò la rilevanza della demercificazione nei suoi studi sulla costruzione del regime socialdemocratico di welfare (Esping-Andersen 1985). Egli sostenne che tale regime era stato forgiato da una classe lavoratrice altamente mobilitata e ben organizzata, che riuscì ad utilizzare il potere politico per superare la sua debolezza nel mercato del lavoro. La rimercificazione consiste nel rovesciamento di tale processo - riducendo le alternative alla partecipazione al mercato del lavoro attraverso un restringimento delle condizioni di accesso o una riduzione dei benefici offerti.

La ri-mercificazione è stata posta al centro delle analisi sulla ristrutturazione dello stato sociale soprattutto da quegli studiosi che vedono nel mondo delle imprese l'attore vincente dell'at- 
tuale periodo storico. Secondo questa prospettiva, la trasformazione del welfare consiste essenzialmente nel ridurre la protezione dei lavoratori nei confronti delle pressioni esercitate dal mercato, costringendo loro ad accettare un'occupazione alle condizioni dettate dal datore di lavoro. Tale formulazione si è rivelata di particolare importanza nelle analisi proposte da studiosi di welfare provenienti dal campo delle relazioni industriali (Clayton e Pontusson 1999; Swenson 1992).

La mercificazione rappresenta evidentemente un'importante dimensione nei recenti conflitti sullo stato sociale e in alcuni casi tale dimensione è stata estremamente rilevante. Gli «incentivi per l'occupazione» sono spesso stati al centro del dibattito, ad esempio nel caso della riforma delle pensioni di invalidità nei Paesi Bassi o dell'abolizione del programma Aid to Families with Dependent Children (Afdc) negli Stati Uniti. Un'analisi ravvicinata di questi esempi evidenzia tuttavia che le riforme non si incentrano sulla ri-mercificazione: anche gli attori che dovrebbero essere particolarmente interessati a questo aspetto ad esempio i datori di lavoro in Germania (Wood in stampa) hanno spesso preoccupazioni più pressanti.

Contenimento dei costi. Nella sua incisiva critica alle analisi delle variazioni del welfare state fondate sullo studio della spesa pubblica, Esping-Andersen ha osservato che i livelli di spesa dipendevano essenzialmente da altri esiti considerati rilevanti dagli attori (la demercificazione, la riduzione della povertà, il mantenimento del reddito, e così via) anziché determinarli. Nella fase di espansione dello stato sociale «è difficile immaginare che qualcuno abbia lottato per conseguire incrementi nella spesa in quanto tale» (Esping-Andersen 1990, 21). Tale affermazione non sembra più corrispondere al vero: il conflitto sul quantum di spesa come tale caratterizza l'attuale fase di austerità. Una serie di pressioni endogene - tra le quali il passaggio dalla produzione industriale a quella di servizi, i mutamenti demografici e familiari, la necessità di far fronte agli impegni di protezione assunti nel passato - convergono nel porre l'accento sugli imperativi di bilancio. La riduzione del deficit pubblico è diventata così la priorità principale in molti dei paesi membri, o aspiranti tali, dell'Unione monetaria europea.

Con l'eccezione di un importante lavoro di Wilensky (1981), gli scienziati sociali hanno per lo più considerato spesa sociale e fiscalità come campi di ricerca distinti. In realtà si trat- 
ta di due facce indissolubilmente legate della stessa medaglia. $\mathrm{Nel}$ contesto attuale, la necessità di finanziare il deficit pubblico rappresenta spesso il principale vincolo pendente sulle politiche sociali. Gli imprenditori e gli interessi finanziari sono preoccupati per gli alti livelli di imposizione fiscale a causa dei loro potenziali effetti sui profitti.

L'altro canale politico di opposizione a un elevato livello di prelievo fiscale, evidentemente, è l'elettorato. Nella misura in cui lo stato sociale si è esteso, le famiglie a medio reddito ne hanno costituito vieppiù le basi finanziarie. Imposte più elevate e più lenta crescita delle retribuzioni reali hanno determinato un diffuso scontento. In molti paesi i politici si trovano a dover affrontare il declino del sistema a ripartizione. Nel corso della golden age essi potevano fare promesse generose differendone $\mathrm{i}$ costi (ad esempio un'alta pressione fiscale). Più che chiedere credito in vista di nuove iniziative, nella fase attuale i politici si comportano invece come esattori che devono far fronte alle promesse del passato. Per quanto quasi ovunque gli elettori si dichiarino ampiamente favorevoli alla spesa sociale, tali sentimenti oggi si uniscono ad una resistenza potenzialmente intensa nei confronti di un incremento significativo del carico fiscale (Bonoli, George e Taylor-Gooby in stampa).

I governi si trovano ad affrontare il poco invidiabile compito di conciliare le spinte a un aumento della spesa pubblica, con potenziali contraccolpi sull'elettorato, con la possibilità che nuove imposte abbiano conseguenze negative sulla performance economica. Nel contempo, molti paesi - in primo luogo quelli soggetti ai criteri di convergenza imposti dall'Unione monetaria europea - si trovano sottoposti a vincoli finanziari e monetari più severi e ciò rende essenziale una strategia di contenimento dei costi.

Modernizzazione. I fini che i sistemi di welfare contemporanei si trovano a dover perseguire non possono ridursi al contenimento dei costi e alla mercificazione; essi includono anche obiettivi riconducibili a un principio di modernizzazione. Per modernizzazione si intendono due ordini di mutamento: la razionalizzazione, che comporta cambiamenti ispirati $\mathrm{da}$ nuove idee su come conseguire i tradizionali obiettivi; l'aggiornamento, che consiste in adattamenti dei vecchi programmi per rispondere a nuove domande e norme sociali.

La razionalizzazione include $\mathrm{i}$ tentativi di modificare pro- 
grammi non più compatibili con la natura degli incentivi o i casi di eccesso di generosità (ad esempio, le pensioni di invalidità nei Paesi Bassi o le pensioni dei dipendenti pubblici in Francia e in Italia). Comprende inoltre riforme complessive, come quella del sistema sanitario, volte a migliorarne l'efficienza nell'erogazione delle prestazioni o nella capacità di risposta alle esigenze e alle domande dei consumatori.

Sul piano analitico, si tratta di un terreno minato. Se nell'attuale contesto le riforme sono spesso disegnate con l'intento di risparmiare, come è possibile distinguere l'impatto delle nuove idee da semplici tagli nell'erogazione delle prestazioni? Evidentemente non si vuole ascrivere alla categoria «assalti allo stato sociale» qualsiasi modifica dei programmi di welfare. Nel lungo periodo, gli attori possono convincersi che alcuni programmi non funzionano come previsto, oppure che è necessario apportare alcune modifiche al fine di ottenere gli obiettivi prefissati, e pertanto eserciteranno pressioni in tale direzione. Le recenti riforme del sistema sanitario svedese, ad esempio, hanno prodotto una considerevole riorganizzazione e una contrazione dell'occupazione, in particolare tra i lavoratori poco qualificati. Per un verso, ciò rappresenta una forte offensiva contro i dipendenti pubblici (Clayton e Pontusson 1999); la riforma sanitaria svedese può essere però letta anche come un tentativo di incrementare la produttività del settore pubblico con l'obiettivo di fornire servizi flessibili e di alta qualità ai consumatori ad un costo politicamente sostenibile. $\grave{E}$ tuttavia difficile affermare che la riforma del settore pubblico equivalga sempre e comunque a una «contrazione» dello stato e del suo impegno nel sociale, giacché non vi sono prove empiriche che mostrino come la riduzione dei costi abbia inciso negativamente sulla qualità del servizio.

L'aggiornamento comprende la modifica dei programmi esistenti o l'avvio di nuovi programmi in risposta a necessità sociali emergenti. Una caratteristica essenziale nella definizione del periodo attuale è la coesistenza di condizioni sociali per molti versi «nuove» con sistemi di welfare sostanzialmente «vecchi» (Esping-Andersen 1999). È stato già osservato che sistemi di welfare maturi tendono ad essere caratterizzati da una certa inerzia, risultante dalle varie forme di path dependence. Inoltre, molti esiti di policy costituiscono effetti ritardati di decisioni adottate molti decenni or sono. Pertanto, vi è una consistente discrasia tra rischi sociali emergenti e ventaglio delle politiche sociali esistenti. 
Per quanto il concetto di modernizzazione sia di per sé problematico, ancor più problematico è ridurre la natura dell'attuale ristrutturazione dei sistemi di welfare a una semplice strategia di contenimento dei costi e di ri-mercificazione. Numerose iniziative, riguardanti, ad esempio, aspetti quali l'equità di genere e l'esclusione sociale, non possono essere incluse in tali categorie.

Rendere pluridimensionale l'analisi della ristrutturazione dei sistemi di welfare consente del resto numerosi vantaggi. In primo luogo, suddividere l'ampia categoria di «mutamento» in sottocategorie più circoscritte affina la nostra capacità di distinguere tra le diverse configurazioni degli esiti. Ė possibile identificare variazioni considerevoli tra varie arene di policy e tra $\mathrm{i}$ vari paesi. Specifici settori dello stato sociale tendono ad essere maggiormente sottoposti a riforme rispetto ad altri. Nel caso della riforma del mercato del lavoro, la ristrutturazione riguarda principalmente gli aspetti di mercificazione e modernizzazione, e ciò dà vita a configurazioni piuttosto interessanti e diverse da un paese all'altro (Wood 1999), mentre il contenimento dei costi costituisce una spinta alla riforma assai meno significativa. D'altro lato, nel caso dei sistemi pensionistici e sanitari, il contenimento dei costi è l'elemento centrale nella maggior parte dei paesi, pur affiancato talvolta da tentativi di modernizzazione. Solo di rado la mercificazione costituisce la dimensione più rilevante per analizzare la natura della riforma in questi settori, cruciali in qualsiasi stato sociale. Nel caso delle politiche per la famiglia, infine, la pressione principale a cui sono sottoposti i sistemi di welfare è l'esigenza di modernizzare le politiche sociali, adattandole a rapporti radicalmente mutati tra mercato, stato e famiglie.

Le distinte dimensioni della ristrutturazione dei sistemi di welfare presentano una diversa salienza non solo da un punto di vista settoriale (tra i diversi programmi di welfare), ma anche sul piano geografico-territoriale (i paesi coinvolti) e temporale. La disaggregazione delle diverse dimensioni di riforma consente infine di descrivere in modo più puntuale gli interessi degli attori e la natura dell'attività politica. Vari attori possono essere interessati a singoli aspetti della riforma o potrebbero avere priorità parzialmente confliggenti. Inoltre, a causa di queste differenze vi potrebbero essere possibilità inattese di legami intersettoriali e scambi negoziati in cui i vari attori rinunciano ad alcune priorità a favore di altre più rilevanti. Uno degli esiti più 
significativi è la formazione di coalizioni inattese, costruite su principi di reciprocità (Bonoli 1999; Rhodes 1999; Myles e Pierson in stampa). Per riprendere lo schema tracciato nel primo paragrafo, riconoscere la pluridimensionalità della riforma è centrale in un contesto in cui molti attori hanno raggiunto un consenso sulla necessità del «mutamento», ma hanno interessi $\mathrm{e}$ priorità diversi.

Tre distinti regimi di welfare. Il secondo fronte di articolazione dell'analisi è connesso all'esistenza di costellazioni politiche e di policy molto diverse (i distinti regimi di welfare). Finora si è parlato essenzialmente di un'unica «logica» di riforma dello stato sociale. Così come è opportuno analizzare le diverse dimensioni della riforma, è altresì necessario rilevare che le politiche di ristrutturazione hanno luogo entro assetti politico-istituzionali tra loro distinti.

La tipologia a tre voci proposta da Esping-Andersen - sistemi di welfare liberale, conservatore e socialdemocratico - ha avuto una notevole influenza nel campo degli studi sullo stato sociale. Nel contempo essa è stata oggetto di numerosi rilievi critici, vuoi perché alcuni paesi erano stati inseriti in una specifica categoria in modo non del tutto convincente, vuoi perché altre dimensioni di variazione erano state trascurate. «Parlare di regime», sostiene Esping-Andersen, «significa indicare che la relazione tra stato e mercato chiama in causa un complesso intreccio di elementi legali e organizzativi» (1990, 2). L'aspetto più promettente dell'analisi di Esping-Andersen consiste nell'affrontare i sistemi di welfare come parte di più complesse configurazioni storiche 4 . I «tre mondi», infatti, non sono il risultato di semplici differenze quantitative in merito ad alcune variabili principali, bensì anche l'esito dell'interazione continua tra vari fattori causali interdipendenti. Gli effetti delle interazioni tendono a prevalere: le variabili possono avere un certo peso solo

4 Sotto questo profilo, l'analisi si svolge in parallelo con un altro filone di indagine assai importante nel campo degli studi comparati di political economy e relativo alle differenze tra i capitalismi contemporanei (Kitschelt et al. 1999; Hall 1999; Soskice 1999). Nell'incisiva analisi di Soskice: «vi sono forti dimensioni di complementarietà interconnesse tra le diverse parti di un assetto istituzionale. Per poter funzionare in modo efficace ciascun sistema dipende dagli altri» $(1999,109)$. Vi sono nuovi importanti sforzi di ricerca, avviati in qualche misura dai contributi di Esping-Andersen, volti ad esplorare le relazioni tra i sistemi produttivi e i regimi di welfare (si vedano Ebbinghaus e Manow (1998), Huber e Stephens (in stampa), Manow (in stampa) e Rhodes (in stampa)). 
se coadiuvate da una serie di fattori aggiuntivi. Tale prospettiva presenta forti affinità con il concetto di «causazione congiunturale complessa» espresso da Ragin (1987).

$\mathrm{La}$ path dependence si colloca in questa stessa prospettiva. $\grave{\mathrm{E}}$ molto probabile che in contesti in cui un insieme di organizzazioni e istituzioni si sviluppano contestualmente per un certo periodo di tempo, rafforzandosi attraverso processi di reciproco adattamento e di selezione competitiva, i processi di path dependence tendano a prevalere. Probabilmente, gli attori organizzativi e istituzionali che non si adattano non riescono a sopravvivere.

Distinte configurazioni di welfare sono prodotte da una causazione congiunturale complessa in cui molti fattori contribuiscono nel tempo a determinare esiti estremamente diversi. Non vi è alcuna giustificazione teorica a sostegno della tesi che ritiene possibile il verificarsi di effetti diretti sugli esiti in caso di una variazione di dieci punti percentuali nel valore di una variabile qualsiasi. Ne è un esempio l'impatto delle istituzioni politiche sulle attuali prospettive di riforma. Secondo Pierson e Weaver (1993), non vi sono chiari presupposti teorici per ritenere che un incremento nella frammentazione istituzionale renda necessariamente più difficoltoso il ridimensionamento dello stato sociale. La concentrazione di autorità include anche l'accountability che potrebbe determinare alcune difficoltà nel perseguimento di politiche impopolari. Tale dinamica di accountability/declinazione delle responsabilità è importante per comprendere le esitazioni in materia di riforma anche da parte di governi unitari, per spiegare le strategie impiegate da tali governi e per mettere in luce la possibilità che un atteggiamento aggressivo possa condurre a un ridimensionamento elettorale.

Nuove prove empiriche dovrebbero mostrare piuttosto chiaramente che la concentrazione dell'autorità politica costituisce una risorsa per chi desidera effettuare una riforma ${ }^{5}$. Partico-

5 Tuttavia, mentre una frammentazione istituzionale può impedire gli sforzi di ridimensionamento, essa può altresì rallentare inizialmente l'espansione dello stato sociale (Swank in stampa). Tale rallentamento si verifica sia a causa di un'influenza diretta della frammentazione istituzionale nell'ostacolare la riforma, sia a causa dei suoi negativi effetti indiretti sulla solidarietà sociale attraverso la promozione di interessi eterogenei. Ciò è in linea con l'assunto generale di molte ricerche di marca istituzionalista secondo cui generalmente gli effetti delle istituzioni non sono univoci e unidimensionali, bensì molteplici e multidimensionali. In altre parole, è necessario distinguere tra effetti causali della frammentazione istituzionale di breve e di lungo periodo. Sul punto si veda Shalev (1998). 
larmente rilevanti sono le esperienze di due casi liberali - Nuova Zelanda e Regno Unito - discussi in dettaglio più avanti. Tuttavia, non è casuale che gli esempi di forte ridimensionamento in assenza di formali veto points istituzionali riguardino sistemi di welfare liberali, in cui gli attori sociali sono disorganizzati e il sostegno dell'opinione pubblica nei confronti dello stato sociale è più limitato che in altri paesi. Sembra altamente improbabile che tale variabile indipendente possa avere analoghe conseguenze in un contesto in cui il sostegno sia più ampio e $\mathrm{i}$ rappresentanti del mondo del lavoro abbiamo maggiori risorse di potere a loro disposizione. La «libertà» di dar vita a riforme radicali deriva non soltanto da un'elevata concentrazione di potere politico, ma anche dalla combinazione di tale fattore con altri aspetti della specifica configurazione politica in cui la riforma viene attuata.

Nell'analisi delle dinamiche della ristrutturazione dello stato sociale è dunque necessario valutare sia la particolare forma di stato sociale, sia lo specifico contesto politico e, più precisamente, la dimensione del sostegno dell'opinione pubblica nei confronti delle prestazioni di welfare e il rapporto tra arena partitico-elettorale e sistemi di intermediazione degli interessi.

\section{La ristrutturazione nei tre mondi di welfare}

Questa sezione presenta un tentativo preliminare di descrivere le distinte politiche di riforma dello stato sociale nei tre «mondi», o regimi di welfare ${ }^{6}$. Mantenere distinti i regimi e disaggregare le dimensioni della ristrutturazione consente di identificare regolarità che non emergerebbero adottando un approccio analitico unitario volto a spiegare un esito specifico (ad esempio, il ridimensionamento) in una serie di casi. Sotto questo profilo, pur utilizzando differenti dimensioni per gli esiti, nell'analisi che segue viene adottata la tipologia individuata da Esping-Andersen. D'altro canto, le dinamiche politiche conside-

6 Sono stati esclusi alcuni casi che talvolta fanno parte di comparazioni analoghe: Grecia, Irlanda, Portogallo e Spagna in quanto sistemi di welfare piuttosto recenti, che alla fine della golden age postbellica si trovavano ancora nel corso di un processo di costruzione dello stato sociale (e presentavano, dunque, problematiche diverse rispetto a quei paesi i cui i sistemi di welfare erano ormai maturi); Svizzera e Giappone, i cui sistemi di welfare non trovano facile collocazione in nessuno dei tre regimi. 
rate sono piuttosto differenti ${ }^{7}$. L'obiettivo è rendere più complessa l'analisi in modo da poter cogliere gli elementi centrali delle diversità rendendo tuttavia egualmente possibile identificare modelli generali.

Il regime liberale. Il primo modello comprende i casi di Australia, Gran Bretagna, Nuova Zelanda e Stati Uniti. In tali paesi gli aspetti più rilevanti della costellazione di welfare sono $\mathrm{i}$ seguenti (Huber e Stephens in stampa): livelli relativamente limitati, in prospettiva comparata, dell'imposizione fiscale e della spesa pubblica; basso livello di occupazione nel settore pubblico; diffusione di programmi di trasferimenti monetari destinati solo a chi supera la verifica di scarsi redditi (income-test); estesa presenza del settore privato in campo pensionistico e dei servizi sociali (quali l'assistenza all'infanzia e l'assistenza sanitaria negli Stati Uniti e in Nuova Zelanda) anche per l'incapacità dello stato sociale di far fronte alle domande di prestazioni sociali.

Le costellazioni politiche presentano diversi elementi comuni. Il primo, e più importante, risiede nella debolezza delle organizzazioni di rappresentanza degli interessi. In prospettiva comparata, si tratta di casi in cui le organizzazioni sindacali mostrano limitate capacità politiche e anche la capacità degli imprenditori di agire collettivamente è limitata. In misura maggiore rispetto agli altri regimi di welfare, la maggior parte dei mutamenti di policy ha un'origine elettorale e partitica.

Di questa dimensione elettorale/partitica è opportuno evidenziare alcuni aspetti comuni. In primo luogo, fino alla recente riforma elettorale neozelandese, in tutti i casi i sistemi maggioritari rappresentavano la norma ${ }^{8}$, determinando forti tendenze verso sistemi bipartitici (o a due partiti e mezzo). Ad eccezione degli Stati Uniti, dove le divisioni tra il Congresso e il Presidente sono ormai consolidate, tali sistemi tendevano a pro-

7 È un aspetto che Esping-Andersen non ha approfondito. Nel suo lavoro del 1990 ha identificato cleavages politici in ciascun regime. Più recentemente (1999) sembra sostenere, anche se in modo non molto elaborato, che «path dependence» ed «elettori mediani» impediscono un radicale mutamento di policy. L'analisi qui proposta si riferisce a distinti cleavages politici nei tre mondi ed è volta a sostenere che difficilmente, pur influenzando in modo determinante le riforme, path dependence ed elettori centrali riusciranno ad impedirle.

8 In Australia vige un sistema elettorale «ordinale». Poiché la seconda scelta dell'elettore ha un certo peso, tale caratteristica induce generalmente i partiti a moderare le proprie posizioni politiche. 
durre governi monopartitici, non senza variazioni circa il grado di controllo esercitato sul governo dalle altre istituzioni politiche. La Nuova Zelanda (fino al 1996) e la Gran Bretagna costituiscono casi puri del «Westminster model» di «dittatura eletta»; i sistemi federali (Australia, Canada e Stati Uniti) presentano invece veto points supplementari, la cui natura e ampiezza variano in ragione del paese, dell'area di policy e del contesto elettorale.

$\grave{E}$ indubbio, inoltre, che il sostegno dell'opinione pubblica nei confronti delle prestazioni sociali pubbliche tenda in questi casi ad essere meno incondizionato che altrove, pur se con considerevoli gradi di variazione interna (Svallfors 1997). E anche la dimensione/capacità politica delle associazioni sindacali, mezzo di sostegno tradizionale, risulta relativamente limitata. L'istituzionalizzazione di alternative di mercato (spesso beneficiarie di sovvenzioni pubbliche) indebolisce infine il senso di attaccamento delle classi medie alle prestazioni di welfare. In molti paesi emerge un elevato livello di disomogeneità nelle percentuali di voto delle classi sociali: basse percentuali di voto espresse da soggetti deboli diminuiscono ulteriormente la loro già limitata influenza politica.

Con ciò non si vuole affermare che in questi paesi la maggioranza degli elettori tenda ad opporsi allo stato sociale. Se li si compara ai paesi socialdemocratici e a quelli conservatori-( $p i$ votal)-corporativi, è probabile che gli elettori centrali siano meno legati alle prestazioni sociali e più inclini ad abbracciare proposte politiche alternative, ad esempio la richiesta di maggiori tagli alla spesa pubblica. I regimi liberali di welfare forniscono pertanto opportunità più numerose ai partiti che intendono coniugare successo politico e una posizione relativamente aggressiva, quando non propriamente ostile, nei confronti di importanti componenti dello stato sociale. Il conflitto politico tra i sostenitori di una ristrutturazione moderata e i sostenitori di un radicale ridimensionamento è quindi più equilibrato di quanto non sia negli altri due regimi.

Tutto ciò si riflette nella configurazione degli esiti. In prospettiva comparata, in Nuova Zelanda e, in minor misura, in Gran Bretagna sono stati effettuati tagli piuttosto consistenti (Stephens, Huber e Ray 1999; Castles e Pierson 1996). Negli Stati Uniti, pur convergenti nella stessa direzione, il mutamento è stato più limitato. Canada e Australia hanno seguito invece una strada piuttosto diversa, segnata da sforzi di contenimento 
dei costi e ri-mercificazione nell'erogazione delle prestazioni di welfare, controbilanciata però dal tentativo di assicurare una protezione per i soggetti in maggiore difficoltà. Per quanto le variazioni tra i paesi negli esiti di policy e nei livelli di disuguaglianza siano piuttosto significative, tali esiti non sono sufficientemente consolidati da consentire ferme conclusioni circa le prospettive nazionali.

Una caratteristica essenziale della ristrutturazione in tutti i sistemi liberali di welfare è stata la priorità accordata alla mercificazione. In tutti questi sistemi i trasferimenti monetari ai soggetti adulti esclusi dalla forza lavoro (le indennità di disoccupazione e l'assistenza sociale) sono stati notevolmente ridotti. I tassi di copertura (in Canada e negli Stati Uniti) e i livelli di assistenza (in tutti i paesi) a favore dei soggetti senza occupazione sono stati drasticamente ridimensionati. In Canada e negli Stati Uniti, inoltre, le garanzie federali sul terreno dell'assistenza sociale sono state abolite. Le autorità statali e substatali si sono attivate con decisione per favorire l'inserimento dei poveri nella forza lavoro, privandoli delle prestazioni offerte dall'assistenza sociale.

In prima analisi, questa caratteristica della riforma dei regimi liberali suscita molte perplessità. Per quale ragione sistemi in cui il peso del mercato nell'erogazione delle prestazioni sociali era già il più accentuato tra tutti $\mathrm{i}$ paesi Ocse hanno spinto in modo ancora più deciso in questa direzione? Una prima risposta risiede nella debolezza politica di chi potrebbe opporsi al processo di mercificazione. Altrettanto importante è il legame che si stabilisce tra sostegno al reddito e mercato del lavoro nelle politiche economiche in cui la flessibilità salariale è considerata il primo rimedio contro alti tassi di disoccupazione. Il deteriorarsi della posizione dei lavoratori poco qualificati sul mercato ha posto i governi di fronte a scelte difficili (Iversen e Wren 1998; Scharpf 1997). In coerenza con i principi fondamentali di un'economia di mercato liberale e «disorganizzata», i paesi liberali hanno implicitamente accettato il fatto che il nuovo contesto richiedesse differenziali salariali più elevati e, in particolare, determinasse un deterioramento delle condizioni salariali per i lavoratori poco qualificati. Tale deterioramento può tuttavia verificarsi soltanto se le opzioni alternative non sono attraenti. In altre parole, la spinta verso la flessibilità richiesta ai lavoratori poco qualificati ha implicato un inasprimento delle condizioni di sostegno al reddito per i soggetti senza lavoro. 
I regimi liberali di welfare hanno condiviso il riorientamento verso la mercificazione, pur se con modalità temporali e gradi di intensità differenti. La dimensione più rilevante del dibattito politico nei sistemi liberali di welfare non ha riguardato l'eventualità che i lavoratori poco qualificati facessero parte del mercato del lavoro oppure no, bensì le condizioni della loro partecipazione. L'aspetto centrale del dibattito attuale concerne dunque l'estensione dell'erogazione dei sussidi che favoriscono la mercificazione. I sostenitori di tale erogazione hanno accettato l'idea di riformare l'assistenza sociale e le indennità di disoccupazione, ma hanno altresì cercato di sostenere il reddito da lavoro, integrando salari al di sotto del livello di povertà offerti da un mercato non regolato con varie forme di prestazioni sociali specifiche. Le argomentazioni a favore di tali prestazioni si ispirano a principi di equità sociale e all'esigenza di effettuare investimenti sociali per favorire lo sviluppo del capitale umano. Molte sovvenzioni sono rivolte a gruppi considerati particolarmente vulnerabili e/o particolarmente bisognosi di sostegno (ad esempio le famiglie monoparentali con figli).

Il pacchetto di integrazioni di reddito varia da paese a paese, ma può includere alcuni dei o tutti i seguenti provvedimenti: incremento delle risorse per il sostegno all'infanzia e delle possibilità di accesso a servizi per l'infanzia a prezzi contenuti; riduzione dei contributi sociali per i lavoratori con salari modesti; incremento del salario minimo'; ampliamento delle possibilità di accesso al servizio sanitario pubblico (negli Stati Uniti) e espansione o introduzione di integrazioni sotto forma di crediti di imposta. Quest'ultimo provvedimento è stato particolarmente rilevante. In tutti i paesi liberali sono state introdotte consistenti integrazioni di reddito sotto forma di crediti di imposta, garantite ad alcune figure di working poors - generalmente famiglie con figli. In particolare, notevole è stata l'espansione dei programmi Earned Income Tax Credit (Eitc) negli Stati Uniti e Family Credit, ora Working Families Tax Credit, in Gran Bretagna. In Australia, le prestazioni a favore dell'infanzia sono $m e$ ans-tested (anche se il 60\% della popolazione continua a beneficiarne), ma l'allargamento del programma Additional Family

9 Nel Regno Unito il governo laburista ha introdotto per la prima volta un salario minimo, il cui importo è rispetto al salario minimo statunitense molto più vicino al salario medio. 
Payment ha fornito alle famiglie di lavoratori risorse supplementari significative rapportate al numero di figli.

Anche il contenimento dei costi ha rappresentato nei paesi liberali una priorità politica. La riduzione del deficit ha costituito un leit motiv e molte forze di centro e centro-sinistra (da Blair, a Clinton, al governo laburista australiano) si sono impegnate a non aumentare le imposte. Tale esito è sorprendente, dati i modesti livelli di spesa pubblica in questi paesi e l'assenza di vincoli esterni analoghi ai criteri imposti dal Trattato di Maastricht. Per quanto nelle tre economie dipendenti dall'esportazione di beni primari (Australia, Canada e Nuova Zelanda) potrebbero verificarsi pressioni esterne per l'ottenimento di un vincolo di bilancio, risulta difficile rilevare tendenze similari in Gran Bretagna e specialmente negli Stati Uniti. Di nuovo, l'approccio basato sul concetto di regime può essere di aiuto per considerare distintamente le strade che conducono a esiti analoghi. L'Italia e gli Stati Uniti possono essere accomunati da una decisa politica volta alla riduzione del deficit di bilancio, ma tale elemento comune può riflettere dinamiche politiche notevolmente diverse. Pur risultando assenti i meccanismi di spinta istituzionale indotti dall'Ume, nei regimi liberali la collocazione dell'elettore centrale, più vicina a posizioni politiche tradizionalmente di destra, sembra facilitare riduzioni della spesa pubblica (e del prelievo fiscale) e del deficit pubblico.

Gli sforzi di contenimento della spesa non sembrano, tuttavia, dar vita ad una risposta liberale differenziata nei settori caratterizzati da un'alta spesa complessiva, quali l'assistenza sanitaria e pensionistica. A differenza delle forme di sostegno al reddito per i soggetti in grado di lavorare, non vi è in questo caso nessun paradigma di policy dominante. Nel settore pensionistico e sanitario, gli esiti sembrano essere fortemente vincolati dai particolari schemi di policy, definiti prima che le domande volte ad ottenere un contenimento dei costi diventassero prevalenti. Nei casi caratterizzati da assetti di policy istituzionalizzati e profondamente radicati, gli elementi di continuità hanno prevalso sulle dimensioni di mutamento. Sul terreno dell'assistenza sanitaria, Australia, Gran Bretagna e Canada hanno compiuto i necessari adattamenti dei loro sistemi con l'intento di ridurre i costi, senza però mettere in discussione i diritti fondamentali a un'assistenza di qualità, e in presenza di un incremento della spesa complessiva. Negli Stati Uniti la più consistente iniziativa di policy è stato il fallito tentativo di allargare la copertura del 
sistema sanitario, che si collocava in ogni caso all'interno di una strategia complessiva di contenimento dei costi. Soltanto in Nuova Zelanda vi sono stati consistenti sforzi per ridefinire in senso restrittivo alcune prestazioni pubbliche nel settore dell'assistenza sanitaria.

Nelle economie di mercato non vi è alcuna volontà esplicita che spinga verso un'espansione del mercato e una contrazione dello stato nel settore dell'assistenza sanitaria. È possibile piuttosto sostenere il contrario: un forte ruolo dello stato nell'assistenza sanitaria è generalmente nell'interesse della maggior parte degli imprenditori. L'iniziativa di riforma dell'amministrazione Clinton era basata su tale presupposto. Probabilmente, il suo fallimento è dipeso più dall'incapacità di un sistema «disorganizzato» di facilitare la negoziazione (Judis 1995) e dall'accumulazione di interessi privati consolidati nell'assetto di policy esistente - accumulazione vincolata da meccanismi di path dependency -, piuttosto che da un riflesso di un inasprimento del conflitto tra imprenditori e consumatori.

Allo stesso modo è evidente che non si è avuta alcuna convergenza nei sistemi pensionistici. Canada e Stati Uniti hanno modificato i loro sistemi consolidati, enfatizzando la dimensione del contenimento dei costi. Non essendo vincolati da precedenti impegni, Regno Unito e Australia hanno intrapreso strade nuove (e molto diverse) per finanziare le prestazioni. La Nuova Zelanda si è dimostrata incapace di procedere in una qualsiasi direzione. In breve, gli effetti esercitati dalla path dependency sono stati molto consistenti in questo settore.

Anche nel caso dei tentativi di mercificazione, le esperienze di Canada e Australia di contenimento dei costi mostrano uno specifico cammino di ristrutturazione. A fronte di una riduzione nella dimensione dei programmi, vi sono stati importanti tentativi di garantire una protezione ai più deboli. In ciascun paese, la strategia dominante incentrata sulla prova dei mezzi ha consentito l'accumulo di risparmi, di cui una parte è stata ridistribuita ai gruppi a basso reddito (Castles 1996; Myles 1996). Tale scambio ha facilitato il mantenimento di un consenso centrista piuttosto ampio nei confronti degli sforzi di risanamento. $\mathrm{Ne}$ è un esempio particolarmente significativo il fatto che in Canada ad una drastica riduzione negli importi erogati a pensionati ad alto reddito si sia accompagnata una consistente espansione del livello delle pensioni minime. Il cambiamento è stato conseguito con un tale successo da consentire al Canada, 
paese caratterizzato da una bassa spesa pubblica, di ridurre $\mathrm{i}$ tassi di povertà degli anziani, portandoli a livelli vicini a quelli scandinavi (Myles 1999).

Le differenze interne al mondo liberale in trasformazione emergono con maggiore chiarezza nelle dimensioni della disuguaglianza salariale e della povertà. I tassi di povertà sono aumentati in misura esponenziale nel Regno Unito e in Nuova Zelanda - e in misura decisamente superiore agli altri paesi Ocse. Sono aumentati in modo meno consistente negli Stati Uniti anche se $\mathrm{i}$ tassi di povertà in questo paese erano più elevati in valore assoluto. In Canada e in Australia, invece, almeno nei primi anni '90 vi sono stati pochi mutamenti. Le politiche volte a proteggere soggetti più deboli dal rischio di sostenere $i$ costi più elevati dell'aggiustamento sono state smantellate in Nuova Zelanda e, fino a tempi recenti, in Gran Bretagna. D'altro canto, in Australia e Canada (e in misura molto più ridotta negli Stati Uniti) tali protezioni sono state in molti casi accresciute al fine di controbilanciare la crescente disuguaglianza nei redditi da lavoro offerti dal mercato.

Il regime socialdemocratico. I casi scandinavi o «socialdemocratici» includono Danimarca, Norvegia, Svezia e (in modo più marginale) Finlandia. Le caratteristiche salienti di questa configurazione di welfare sono ben note: generosi trasferimenti che coprono un'ampia gamma di rischi e garantiscono un alto livello di copertura; servizi sociali pubblici estremamente numerosi in prospettiva comparata; un insieme di politiche a sostegno delle famiglie e del mercato del lavoro che determina tassi molto alti di partecipazione della forza lavoro maschile e femminile. Infine, la dimensione delle prestazioni pubbliche ha riempito spazi altrove occupati dal mercato: infatti, l'erogazione di servizi sociali da parte di privati, ad esempio le pensioni, resta estremamente limitata.

La costellazione politica in questi paesi comprende una serie di aspetti che creano forti coalizioni favorevoli alle politiche di welfare. La densità sindacale è elevata e le associazioni sindacali hanno mantenuto robusti legami con forti partiti socialdemocratici. In prospettiva comparata, i sindacati del settore pubblico presentano dimensioni estremamente ampie. Le donne sono altamente mobilitate e legate alle politiche sociali pubbliche (che peraltro costituiscono la fonte primaria dell'occupazione femminile e forniscono un supporto essenziale alla partecipazio- 
ne femminile nel mercato del lavoro). Dato tale assetto istituzionale, non sorprende che l'opinione pubblica rimanga fortemente favorevole agli aspetti più salienti dello stato sociale socialdemocratico (Svallfors 1995; 1997; Andersen 1997; Rothstein 1998). I sistemi politici di questi paesi consentono controlli limitati sulle maggioranze parlamentari, ma la frequenza di governi di coalizione rende l'autorità politica meno concentrata rispetto agli assetti ispirati al modello di Westminster. Inoltre, i governi operano in un contesto in cui le organizzazioni imprenditoriali e sindacali sono diffuse e ben organizzate. Tali organizzazioni sono al contempo possibili controparti di eventuali negoziazioni e forti oppositori politici in caso di dissensi.

Il risultato principale della ristrutturazione dello stato sociale nei paesi socialdemocratici riflette un contesto che garantisce un sostegno molto ampio per le prestazioni sociali pubbliche. La sfida principale che tali sistemi hanno dovuto affrontare è stato l'adattamento all'incremento dei tassi di disoccupazione. I sistemi di welfare socialdemocratici si trovavano in equilibrio fiscale con un livello di disoccupazione pari al $3 \%$, ma a causa della generosità dei trasferimenti si trovarono in difficoltà quando la disoccupazione cominciò a crescere in misura consistente. Quando fu chiaro che l'incremento sarebbe stato permanente, venne introdotta una combinazione di tagli alle prestazioni, riforme volte a produrre risparmi nel settore delle politiche sociali (in particolare, nel settore sanitario) e inasprimenti fiscali. Le variazioni tra i singoli paesi nelle modalità temporali e implementative della ristrutturazione riflettono i tempi e la portata della crisi economica e il grado di adattamento necessario per ristabilire l'equilibrio.

Prima di tracciare le principali dinamiche della riforma, è necessario fare cenno a ciò che si può definire l'interpretazione ortodossa delle politiche di welfare nelle socialdemocrazie contemporanee. Essa attribuisce una forte centralità alle differenze tra i settori «esposti» alla o «protetti» dalla competizione internazionale. Esping-Andersen $(1990 ; 1996)$ considera tale dimensione come la linea predominante di cleavage nei paesi socialdemocratici (cfr. anche Swenson 1992; Clayton e Pontusson 1999; Iversen 1998).

Le prove empiriche a sostegno di tale interpretazione sono tuttavia, ad essere generosi, estremamente limitate. Anche nei paesi socialdemocratici in cui si può ipotizzare che ampi settori pubblici possano generare effetti analoghi, convinti sostenitori 
come Clayton e Pontusson sono costretti a riconoscere che le prove empiriche sono modeste ${ }^{10}$. Nel suo recente contributo volto a stabilire se i cleavages ipotizzati con riferimento allo stato sociale sono presenti nell'opinione pubblica, Svallfors conclude che «... la distinzione tra occupazione nel settore pubblico e occupazione nel settore privato non sembra costituire una linea di demarcazione particolarmente significativa» (1997, 292).

Quali sono le ragioni che spiegano la mancanza di prove empiriche a sostegno di ipotesi da più parti sostenute? Le risposte servono per comprendere molti aspetti dell'attuale regime socialdemocratico e contribuiscono a fornire elementi più solidi per analizzare la sua attuale politica. In primo luogo, l'assunto che i lavoratori «produttivi» debbano entrare in conflitto contro i lavoratori «improduttivi» del settore pubblico si fonda su un'interpretazione piuttosto discutibile del ruolo dei lavoratori del settore pubblico nelle economie socialdemocratiche. Pur essendo spesso implicite, le spiegazioni di tale cleavage presentano più affinità con opinioni sul settore pubblico diffuse in ambienti fortemente neoclassici che considerano tale settore dominio di pratiche di rent-seeking. Ad esempio, in tale prospettiva un lavoratore del settore pubblico impiegato nell'assistenza nei confronti di soggetti alcolizzati o di bambini disabili è sostanzialmente un parassita rispetto agli elementi produttivi della società. In quest'ottica, sarebbe lecito aspettarsi che, con il trascorrere del tempo, gli elementi produttivi rifiutino la diffusione di rendite e cerchino di ridimensionare il settore pubblico.

Invece, vi sono numerosi motivi per sostenere che vi siano benefici effetti economici indotti dagli ampi programmi di prestazioni sociali in un regime socialdemocratico. Molti di questi programmi sono concentrati sullo sviluppo del capitale umano. Politiche attive del lavoro, formazione e attività educative forniscono un solido sostegno allo sviluppo delle competenze che, dal canto loro, contribuiscono al mantenimento di alti livelli di

10 Anche tali prove sono state oggetto di numerose critiche (Lindbom 1999). In Svezia, paese studiato da Clayton e Pontusson, vi sono segnali limitati di un ridimensionamento dei servizi sociali, compiuto con l'impiego di trasferimenti monetari. In alcuni casi, sembra effettivamente che i servizi abbiano conosciuto un'espansione. In altri, in particolare nel sistema sanitario, vi sono state contrazioni dei livelli di occupazione, che tuttavia sembrano da collegarsi a incrementi di efficienza e non tanto alla diminuzione della qualità del servizio. 
qualità della vita. Almeno altrettanto significativo, tuttavia, è il successo di questo regime nella capacità di sostenere il desiderio delle donne di combinare le attività familiari (inclusa la cura dei figli) con una partecipazione attiva nella vita economica. A fini descrittivi, gli economisti spesso considerano gli outputs dei servizi pubblici in modo analogo agli inputs. È invece necessario distinguere la nostra incapacità o il nostro disinteresse nella misurazione della produttività o dell'output dalla reale mancanza di produttività/output. Le rilevazioni empiriche degli atteggiamenti dell'opinione pubblica continuano a mostrare che nei paesi socialdemocratici la maggior parte degli elettori percepisce i servizi sociali pubblici come un importante contributo alla qualità della vita.

Si può evidenziare anche un secondo aspetto problematico concernente il cleavage pubblico/privato. E curioso che in un'analisi incentrata sulle classi o su segmenti di classi l'interesse rivolto alle alleanze interclassiste sia largamente avulso dal contesto sociale. I lavoratori del settore privato, e le loro controparti del settore pubblico, vengono considerati monadi isolate. $\grave{E}$ noto, invece, che $\mathrm{i}$ mercati del lavoro in questo regime sono ampiamente stratificati per suddivisione di genere, giacché i lavoratori di sesso maschile sono notevolmente più numerosi nel settore privato, mentre la forza lavoro femminile è concentrata nel settore pubblico. Tale cleavage politico, quindi, esiste probabilmente in molte famiglie. Se un lavoratore del settore privato è sposato con una lavoratrice del settore pubblico ed essi mandano i loro figli in asili pubblici, qual è la più plausibile configurazione di coalizioni? In questo caso, risulta estremamente rilevante introdurre le dimensioni di genere e della struttura familiare negli approcci di politica economica. Tale aspetto mostra perché alcune delle più diffuse argomentazioni a favore della ristrutturazione dello stato sociale possono essere molto più deboli rispetto a quanto non si pensi comunemente.

Se l'agenda di policy non comporta la riduzione della forza lavoro impiegata nel settore pubblico, quali sono stati gli argomenti utilizzati per sostenere le pressioni di ristrutturazione nei paesi socialdemocratici? Essi si concentrano prevalentemente sulle dimensioni di contenimento dei costi e di modernizzazione dei programmi. Invece, generalmente, la ri-mercificazione ha rappresentato una priorità meno importante. Tale aspetto riflette il fatto che, mentre la socialdemocrazia ha tradizionalmente svolto una funzione «demercificante», ciò è avvenuto in modo 
tale da produrre contestualmente alti tassi di partecipazione nel mercato del lavoro e alti tassi di occupazione. Se incentivare gli individui ad inserirsi nel mercato del lavoro è sempre stato un obiettivo implicito nel modello socialdemocratico, mutevoli sono le condizioni in cui ciò si è verificato. Benché la tendenza che recentemente diversi riformatori svedesi hanno chiamato work-line (chi è nelle condizioni di poter lavorare, dovrebbe farlo), tali mutamenti hanno in genere accentuato nel modello socialdemocratico le aspettative, già tradizionalmente forti, dell'adozione di interventi a favore dell'occupazione.

Un'eccezione è costituita da specifici programmi che, a causa delle condizioni del nuovo mercato del lavoro o a causa di problemi generati dai programmi stessi, hanno determinato reali ostacoli nell'incentivazione al lavoro. Nei casi in cui i programmi si svolgevano in modo inadeguato, sono state introdotte numerose riforme per ridurre $\mathrm{i}$ benefici connessi alle indennità di malattie di breve durata e per migliorare il monitoraggio dei beneficiari. Come è stato osservato da Stephens (1996), tali riforme erano già state completate prima dell'incremento della disoccupazione: i decisori sembravano già essere convinti della problematicità di specifici programmi che, invece di proteggere dai rischi legati a problemi di salute, incoraggiavano l'assenteismo. Pertanto, vi sono forti prove a sostegno dell'ipotesi che il mutamento di policy sia dovuto ad un'esigenza modernizzante (rafforzata da pressioni volte al contenimento dei costi) e non alla mercificazione delle prestazioni sociali.

Tuttavia, sotto molti profili i paesi socialdemocratici hanno avuto una necessità minore di concentrare $\mathrm{i}$ propri sforzi su processi di modernizzazione - in particolare se confrontati con il regime democratico-cristiano. Il regime socialdemocratico è caratterizzato da tassi molto elevati di partecipazione nella forza lavoro sia maschile che femminile; contribuisce al mantenimento di tassi di fertilità piuttosto elevati; possiede un'ampia esperienza nel campo delle politiche attive del lavoro volte a limitare l'esclusione sociale e a incrementare il livello delle competenze professionali; infine, non contiene alcuna dimensione clientelare simile a quelle rilevate in gran parte dei paesi appartenenti al regime conservatore.

Il regime conservatore. Il terzo raggruppamento di paesi, definiti indifferentemente conservatori, continentali, democraticocristiani o bismarckiani, include Austria, Belgio, Francia, Ger- 
mania, Italia e Paesi bassi. Le configurazioni di welfare di questi paesi generalmente comprendono: alti livelli di spesa pubbli$\mathrm{ca}$, in particolare pensionistica; un numero limitato di dipendenti pubblici; un ampio sostegno nei confronti di schemi di previdenza anticipata o programmi accomodanti di protezione dalla disoccupazione e dall'invalidità (Ebbinghaus 1999); elevati livelli di tassazione e presenza di politiche esplicite o implicite per la famiglia (inclusi modalità di tassazione, assenza di programmi di assistenza infantile e debole sostegno nella crescita del settore dei servizi) che disincentivano la partecipazione femminile alla forza lavoro. Tali elementi sono legati ad una struttura bismarckiana di incentivi, volti al mantenimento dello status acquisito e in molti casi a separati fondi categoriali (spesso diversi tra loro). I sindacati e gli imprenditori tendono a svolgere un ruolo molto significativo nella gestione di questi fondi. In alcuni paesi (ad esempio, l'Italia), i regimi conservatori di welfare hanno costituito solide basi per l'adozione di politiche sociali clientelari (Ferrera 1996).

Le costellazioni politiche sono spesso diverse, ma è possibile evidenziare alcuni tratti comuni che rendono i regimi conservatori piuttosto differenti dagli altri. Per quanto non tutti questi paesi possano essere definiti corporativi (è il caso della Francia), i sistemi organizzati di intermediazione degli interessi sono generalmente importanti: «in Europa, quattro lavoratori su cinque ricevono salari che riflettono l'esito di un processo di contrattazione collettiva» (Golden, Wallerstein e Lange 1999, 204). Tuttavia, tali sistemi differiscono nella misura in cui i partner sociali sono intenzionati a, e in grado di, negoziare numerosi programmi dello stato sociale. Al contempo, in paragone con i casi socialdemocratici, i sindacati del settore pubblico sono generalmente di dimensioni limitate e politicamente deboli.

I sistemi elettorali di questi paesi tendono a generare molteplici veto points. L'uso del voto proporzionale (seppur mitigato in alcuni paesi) rende molto rara la presenza di governi monopartitici. Il federalismo (Germania e, sempre più, Belgio) e il ruolo istituzionalizzato dei partner sociali spingono verso strategie di riforma negoziata. Un ultimo elemento dell'assetto politico istituzionale degno di attenzione risiede nel fatto che tutti questi paesi sono membri dell'Unione monetaria europea e quindi vincolati dai criteri di Maastricht.

Anche i sistemi di partito e l'opinione pubblica hanno favorito il consolidamento di un sostegno politico nei confronti del 
welfare state. Pur restando generalmente rilevanti, i partiti socialdemocratici sono molto più deboli rispetto ai paesi scandinavi. Il centro-destra dello spettro politico di questi paesi è sempre stato dominato dai partiti cristiano-democratici che hanno avuto un ruolo importante e talvolta determinante nella costruzione di tali modelli di welfare (Van Kersbergen 1995). Pur contenendo correnti interne piuttosto critiche nei confronti di ampie garanzie sociali, in generale questi partiti sono decisi a lasciare immutate le principali caratteristiche del contratto sociale. Date le dimensioni di questi sistemi di welfare, non sorprende che l'opinione pubblica sia largamente favorevole al loro mantenimento (Svallfors 1997). Tale favore è tuttavia minore rispetto ai paesi scandinavi e presenta una maggiore ambiguità, dovuta alla presenza di vistose disuguaglianze di trattamento, spesso associate alla dimensione clientelare delle prestazioni sociali (Ferrera 1997).

Gran parte degli osservatori concorda sul fatto che i paesi continentali si trovano di fronte a pressioni di aggiustamento degli assetti di welfare senza precedenti nella loro storia (Esping-Andersen 1996; 1999; Scharpf 1997). Esping-Andersen ha elaborato una diagnosi convincente dei seguenti problemi interconnessi: costi elevati $\mathrm{e}$ in aumento dovuti a prestazioni pensionistiche generose e a uscite incentivate dal mercato del lavoro; bassa partecipazione femminile al mercato del lavoro; elevati tassi di disoccupazione tra i lavoratori non qualificati (unitamente ad alti costi fissi del lavoro dovuti all'elevata dimensione dei contributi sociali); bassi tassi di fertilità.

Nell'attuale contesto economico e sociale, gli alti carichi contributivi che gravano sul costo del lavoro - indirizzati prevalentemente al finanziamento delle pensioni - frenano tanto lo sviluppo dell'occupazione nel settore privato (creando livelli salariali minimi elevati) quanto l'occupazione nel settore pubblico (a causa del sovraccarico fiscale). Disoccupazione e stagnazione dei tassi di partecipazione della forza lavoro restringono a loro volta la base fiscale disponibile per il finanziamento delle pensioni e creano pressioni politiche per l'incentivazione dei pensionamenti anticipati attraverso strumenti di politica sociale, in particolare prepensionamenti e pensioni di invalidità. Tutti questi paesi inizialmente hanno risposto ai problemi di occupazione facilitando l'uscita dal mercato del lavoro con specifici programmi (Kohli et al. 1991; Ebbinghaus 1999). La stagnazione occupazionale e la necessità sempre più consistente di trasferi- 
menti hanno determinato ulteriori inasprimenti fiscali. Di fronte a tali difficoltà, il blocco dello sviluppo del settore pubblico e privato dei servizi ha indebolito la capacità delle donne di partecipare alla forza lavoro e di curare i figli, contribuendo al declino dei tassi di fertilità che a sua volta indebolisce l'equilibrio fiscale di lungo periodo. Il problema, infatti, non risiede esclusivamente nel fatto che questi paesi devono affrontare forti pressioni di aggiustamento; risiede anche nella possibilità reale di raggiungere un equilibrio caratterizzato da un tasso di occupazione ridotto e da un basso tasso di fertilità. Nella sintesi a tinte fosche espressa da Esping-Andersen, «questi sistemi si trovano intrappolati in una spirale negativa che si autorafforza» $(1996,68)$.

I paesi del regime continentale si trovano di fronte a due agende di riforma distinte ma complementari. Primo, vi è la necessità di espandere le opportunità di impiego, che nel mondo postindustriale significa incrementare l'occupazione nel terziario. Tale riforma incorre in una serie di ostacoli interconnessi, che, ad esempio, rendono difficili gli sforzi da parte delle donne di combinare il lavoro retribuito e la cura dei figli. I già alti tassi di imposizione fiscale rendono difficilmente praticabile la strada socialdemocratica, caratterizzata da un'espansione dell'occupazione nel settore dei servizi sociali; alti carichi fiscali fissi e salari di ingresso limitano la crescita del settore privato dei servizi, in particolare per servizi caratterizzati da una bassa produttività e, quindi, da bassi salari (Scharpf 1997).

La seconda agenda di riforma consiste nel conseguire un contenimento dei costi nei principali programmi sociali - pensioni, sanità e invalidità. I sistemi continentali sono incentrati su consistenti flussi di trasferimenti e, nelle forme più estreme (ad esempio in Italia e in misura ridotta in Francia e Germania) sembrano più dei «pension states» che veri e propri welfare states. Alti livelli di imposizione fiscale riducono il grado di tolleranza pubblica, rendendo inaccessibili nuovi spazi fiscali volti al conseguimento di una qualche espansione dei servizi sociali. Più specificamente, alti contributi sociali spingono verso l'alto $\mathrm{i}$ costi (fissi) del lavoro per le imprese, riducendo le prospettive di crescita dell'occupazione offerte dal settore privato. Pertanto, il successo nel perseguimento delle proposte di contenimento dei costi contribuirebbe all'obiettivo di ampliare l'occupazione nel settore dei servizi.

Una strategia di contenimento dei costi e una riforma del 
mercato del lavoro rappresentano evidentemente sfide politiche considerevoli. Ampi programmi di sicurezza sociale danno origine a forti interessi consolidati e seri sforzi volti alla riduzione delle spese attraverso una minore garanzia di benefici o il restringimento dei criteri di eleggibilità possono pertanto incidere su blocchi elettorali molto ampi. Sono questi tipi di riforme ad aver causato grandi manifestazioni pubbliche in Francia, Italia, Germania e Paesi bassi. E i governi che hanno avviato tali riforme (Juppé in Francia, numerose coalizioni nei Paesi bassi, Berlusconi in Italia) hanno successivamente pagato un alto prezzo in termini elettorali per le loro iniziative indirizzate a un ridimensionamento del welfare. Inoltre, il tentativo di aprire il mercato del lavoro a lavoratori poco remunerati può suscitare un'aperta contestazione delle parti sociali. E non dovrebbe essere trascurato il fatto che tali contestazioni possono provenire non soltanto dalle associazioni sindacali, ma anche dagli imprenditori: essendosi adattati a molti aspetti di un regime ad alti salari ed elevati livelli di competenze professionali, e avendo beneficiato di contributi pubblici per ridurre il costo del lavoro, è possibile che anche gli imprenditori resistano a, o sostengano solo parzialmente, le riforme del mercato del lavoro.

Esping-Andersen (1990) ha descritto il nuovo cleavage presente nel regime conservatore come una frattura tra insiders e outsiders. Tuttavia, così come nelle analisi del regime socialdemocratico, è probabile che un semplice approccio di political economy incorra nell'errore di sopravvalutare il significato politico di cleavages che dovrebbero aver luogo all'interno di moltissime famiglie. Gli outsiders (ad esempio, donne disoccupate e giovani) spesso dipendono economicamente dagli insiders, e ciò potrebbe di fatto intensificare le resistenze a qualsiasi mutamento considerato minaccioso per il regime di welfare esistente.

Ciononostante, è in atto una decisa politica di ristrutturazione all'interno del regime continentale, ben riassunta nell'idea di nuovo centro (new middle o neue Mitte) cara a Blair e Schroeder. Tale prospettiva, le cui origini sono state esposte nella prima sezione di questo lavoro (i sistemi di welfare generano forte sostegno e, contemporaneamente, si trovano di fronte a consistenti pressioni esogene), sembra essere particolarmente appropriata per l'analisi del regime continentale. Nei paesi continentali il conflitto politico dominante non è tra insiders e outsiders, bensì tra chi cerca di creare le basi per un sistema di prestazioni sociali moderno, fiscalmente e politicamente sostenibile, e i 
soggetti che appoggiano il mantenimento dello status quo (strategia dell'immobilismo) oppure un più consistente programma di liberalizzazione (strategia dello scontro).

L'issue politica più saliente nell'Europa continentale nel corso degli ultimi venti anni ha riguardato la domanda se i singoli paesi fossero o meno in grado di sviluppare la loro capacità di avviare e di appoggiare coalizioni di «nuovo centro» nella ristrutturazione dello stato sociale. Sotto il profilo dei risultati, vi è consenso sul fatto che i Paesi bassi rappresentino un caso di successo della riforma (il c.d. miracolo olandese). In un'ipotetica classifica delle ristrutturazioni di welfare di maggior successo, l'Italia si colloca al secondo posto, mentre Belgio, Francia e Germania rappresentano casi di «welfare ibernato», per aver esibito fin qui capacità di riforma molto limitate ${ }^{11}$. Particolare risalto è stato accordato al contrasto tra il successo olandese e il fallimento tedesco (Hemmerijck e Manow 1998). Da tali esercizi comparati resta invece di solito esclusa l'Austria: la sua buona performance economica - e in particolare la sua capacità di contenere il tasso di disoccupazione - ha infatti circoscritto, almeno fin qui, le pressioni a favore di una ristrutturazione del sistema di welfare.

Come ho cercato di mostrare nelle pagine precedenti, è molto difficile riuscire ad individuare regolarità che tengano conto dei risultati delle riforme adottate in tempi diversi nei vari paesi. Un'analisi sistematica che proceda in tale direzione non rientra negli obiettivi di questo lavoro. Mi sembra però importante volgere brevemente l'attenzione a due interpretazioni ampiamente condivise, che a me sembrano erronee in maniera esemplare. Si tratta, da un lato, del giudizio eccessivamente generoso che accompagna i risultati ottenuti dalle coalizioni riformiste nei Paesi bassi e in Italia e, d'altro lato, dell'eccessiva severità di giudizio nei confronti del caso tedesco, descritto come un sistema di welfare intrappolato in una politica dello status quo.

Considerati come «casi disperati» fino a poco tempo fa, l'Italia (Ferrera e Gualmini 1999 e 2000; Baccaro e Locke 1996) e i Paesi bassi (Visser e Hemmerlijck 1997) hanno recentemente intrapreso una incisiva azione di risanamento. Ridimensiona-

11 L'analisi di Levy (1999) presenta invece toni più ottimistici, ma egli stesso è costretto a riconoscere che la sua rassegna dei mutamenti di policy è altamente selettiva, volta più specificamente a gettare luce su un particolare stile di riforma che non a fornire una descrizione sistematica del processo complessivo di ristrutturazione. 
re la portata del «miracolo» non significa naturalmente negare il significato degli sforzi di ristrutturazione compiuti in entrambi i paesi. Per illustrare un dato significativo: in Italia le politiche esistenti prima delle riforme del 1992, 1996 e 1997 davano in proiezione una spesa pubblica pensionistica pari al $23 \%$ del Pil nel 2035; proiezioni più recenti stimano che la spesa toccherà le punte più alte a un livello leggermente inferiore al $16 \%$ (rispetto all'attuale $14 \%$ ) nel 2030, per poi iniziare a ridursi (Ferrera e Gualmini 1999, tab. 1). Nei Paesi bassi, le politiche di riforma del mercato del lavoro e le riduzioni salariali negoziate hanno determinato un forte incremento dell'occupazione, in particolare a tempo parziale. Molti sforzi sono stati fatti, con risultati ancora incerti, per raggiungere l'obiettivo di una minore dipendenza dalle pensioni di invalidità e dai prepensionamenti. Ne è un esempio la ristrutturazione dei programmi di invalidità, che ha notevolmente incrementato il ruolo di controllo dello stato.

Un'analisi comparata dei rendimenti delle riforme dipende in misura consistente dalla scelta della scala temporale di riferimento. Se consideriamo i casi dell'Italia e dei Paesi bassi dal 1973, considerato l'anno in cui ha termine la golden age, le valutazioni possono essere molto differenti. In entrambi i casi, la situazione è inizialmente peggiorata a causa della scelta di espedienti di breve periodo escogitati dai policy-makers, incluse un'espansione programmatica della spesa, una politica di deficit spending e l'incentivazione dell'uscita dal mercato del lavoro. In larga misura, le politiche seguite a partire dal 1982 nei Paesi bassi e dagli anni '90 in Italia si sono focalizzate invece su un'attività di riduzione dei danni causati negli anni precedenti. Nonostante il c.d. «miracolo», nei Paesi bassi i tassi di partecipazione al mercato del lavoro sono tuttora inferiori a quelli tedeschi. Gran parte dell'espansione, fortemente auspicata, dei tassi di partecipazione femminile sembra tradursi in lavori a tempo parziale estremo. Calcolando il totale delle ore retribuite nel mercato del lavoro, i tassi relativi alle donne nei Paesi bassi si collocano all'ultimo posto nella classifica dei paesi dell'Unione europea (Daly 1998). L'Italia, nonostante le riforme, continua ad avere il più elevato livello di spesa pensionistica al mondo, un debito pubblico molto consistente e tassi di partecipazione al mercato del lavoro e di fertilità estremamente bassi.

Inoltre, è necessario fornire un'interpretazione più sfumata del caso tedesco, spesso considerato come un caso di aggiusta- 
mento fallito (Manow e Seils 1999). Gli sforzi di riformare il mercato del lavoro si situano in Germania in una direzione che la teoria riesce facilmente a spiegare. In un sistema politico costellato di veto points, i tentativi di riforma hanno di norma fallito nel proposito di superare la resistenza degli interessi organizzati, fortemente radicati nell'esistente configurazione del mercato del lavoro. Tuttavia, un altro aspetto del welfare tedesco è stato sottoposto a notevoli aggiustamenti, in particolare in merito al contenimento dei costi: per quanto lenta e difficile sia stata la riforma sanitaria, essa è stata compiuta. Nei primi anni ' 90 una coalizione informale comprendente i due maggiori partiti e le associazioni imprenditoriali e sindacali è stata in grado di imporre un significativo contenimento dei costi, unitamente all'introduzione di ticket modesti. Due grandi processi di scambio, entrambi rilevanti momenti di modernizzazione, hanno reso la ristrutturazione dolorosa. Le riforme hanno ridotto le disuguaglianze tra i vari fondi a tutela della malattia (un tradizionale obiettivo dei sindacati) e allocato una consistente quota delle risorse finanziarie in un nuovo programma di sicurezza sociale.

Nel caso delle pensioni, dalla metà degli anni '70 si sono verificati numerosi e considerevoli mutamenti volti al contenimento dei costi. Ciascuna riforma ha richiesto ampie negoziazioni tra i maggiori partiti e le parti sociali ${ }^{12}$. Come è stato indicato da Alber (1998), la maggiore conseguenza di tutti questi tentativi non è stata la riduzione dei livelli di spesa pensionistica o delle prestazioni erogate ai pensionati, bensì l'eliminazione del considerevole aumento dei costi pensionistici previsti dalle precedenti politiche pensionistiche. Le prestazioni pensionistiche sono diminuite di circa il $30 \%$ rispetto al livello che avrebbero raggiunto se non fossero state apportate ripetute modifiche alle politiche in vigore alla metà degli anni ${ }^{\prime} 70^{13}$. Il $50 \%$ circa dei risparmi è stato ottenuto con le politiche del governo Spd-Fdp in carica fino al 1982, e il restante $50 \%$ è attribuibile ai governi successivi guidati da Kohl. Sforzi indirizzati verso un conteni-

12 Almeno in un caso, gli imprenditori e i sindacati hanno sottoposto una proposta congiunta alla Commissione incaricata di elaborare il progetto di riforma.

13 Il caso tedesco illustra l'ampiezza delle riduzioni possibili nella politica pensionistica quando i policy-makers perseguono obiettivi di lungo periodo, ottengono un ampio consenso tra le élites e non restringono in modo eccessivo il valore reale delle prestazioni erogate ai beneficiari. 
mento dei costi si sono avuti anche in altri programmi di welfare, anche se Alber mostra che i più deboli (ad esempio, le famiglie dipendenti dai servizi dell'assistenza sociale) hanno ricevuto un trattamento relativamente favorevole.

La ristrutturazione tedesca è stata poco considerata non soltanto perché è stata poco significativa in alcuni settori (in particolare, per ciò che riguarda il mercato del lavoro), ma anche perché non è stata traumatica. Inoltre, la portata dei mutamenti è stata particolarmente messa in ombra dall'enorme impatto dell'unificazione tedesca, motivo di un forte squilibrio fiscale. Tuttavia, di fronte a queste circostanze avverse (in larga misura inevitabili, a differenza di quanto avvenuto nei Paesi bassi e in Italia) la Germania è riuscita a reagire in modo piuttosto positivo. È riuscita sostanzialmente a non ritoccare la percentuale del Pil impiegata per la spesa sociale, a non ridurre le prestazioni per $\mathrm{i}$ poveri, introducendo al tempo stesso alcuni importanti elementi di modernizzazione nella gestione degli strumenti più rilevanti del sistema di welfare.

A mio avviso, quindi, Germania, Italia e Paesi bassi dovrebbero essere tutti e tre classificati come esempi di ristrutturazioni piuttosto significativi. Ciascun paese ha unito un certo contenimento dei costi con sforzi volti al conseguimento di una modernizzazione del sistema di welfare. I percorsi di riforma sono stati differenti: Paesi bassi e Italia si sono trovati alle prese con un «sovraccarico» di problemi originato dalle crisi economiche e hanno quindi dato vita a coalizioni politiche impegnate in direzione riformista; in Germania, la riforma è stata invece più lineare e incrementale. Questi tre casi sono in contrasto con le esperienze di Francia e di Belgio, dove le iniziative di ristrutturazione sono state in generale più limitate.

Nei casi in cui la riforma è stata più consistente, la dinamica politica ha determinato la costruzione di una coalizione di «nuovo centro» (new middle). Le configurazioni politiche esistenti hanno richiesto un accorto negoziato tra tutti (o quasi) i più importanti attori politici - non soltanto per superare i veto points, ma anche per raggiungere un livello di legittimità sufficiente a far sostenere i tagli e a garantire un'implementazione efficace. Nei Paesi bassi e in Italia tale processo è stato evidente: ampie coalizioni centriste sono riuscite a convincere attori chiave, inclusi gli elettori, della necessità della riforma, e hanno introdotto consistenti innovazioni; numerosi sforzi sono stati intrapresi al fine di rassicurare che l'obiettivo dei riformatori era 
la salvezza e non lo smantellamento del welfare; le riforme di politica sociale si sono concentrate sui programmi più dispendiosi (pensioni in Italia, invalidità nei Paesi bassi) che stavano compromettendo l'efficienza del sistema e diventavano più difficili da giustificare. Date le difficoltà fiscali esistenti in entrambi i paesi, le opportunità per meccanismi di scambio politico erano limitate, ma l'esplicita volontà di ampliare aspetti sottodimensionati delle prestazioni sociali ha reso le riforme più accettabili. Infine, in ciascun paese sono stati compiuti considerevoli sforzi per sostenere i più deboli; finora, al consistente contenimento dei costi non si è accompagnato un incremento significativo nei livelli di povertà.

Anche in Germania questi successi riflettono l'utilizzo dei metodi del «nuovo centro» per la negoziazione tra le élites e l'ottenimento di una mutua legittimazione. I due partiti più importanti, unitamente alle parti sociali, hanno partecipato in modo attivo e proficuo al confronto sulle riforme da adottare. La peculiarità tedesca, invece, risiede nel carattere informale, intermittente e settoriale del processo di riforma. A differenza dei Paesi bassi e dell'Italia, la riforma si è sviluppata in modo incrementale, e non in seguito ad un'unica e ben propagandata campagna di mutamento complessivo.

Il compito principale di chi studia il regime conservatore è stabilire quali fattori rendano possibile la formazione di ampie coalizioni centriste. Per ora è possibile soltanto cercare di definire un insieme sufficientemente convincente di ipotesi. In primo luogo, si può distinguere tra fattori che influiscono sulla richiesta di una risposta politica negoziata e fattori che influiscono sulla capacità del sistema di dare vita a tale risposta. Dal lato della domanda, più specificamente, vi sono condizioni che convincono gli attori rilevanti dell'impraticabilità di strategie di immobilismo o di scontro frontale. Da questo punto di vista, emarginare i sostenitori di opzioni fortemente conflittuali spesso facilita il raggiungimento dell'obiettivo di riforma. Date le peculiari caratteristiche del regime conservatore, è inoltre probabile che la propensione a consentire una piena liberalizzazione sia scarsa. Anche nel caso dei soggetti che in linea di principio potrebbero considerare auspicabile tale strategia, $i$ segnali in tal senso provenienti dall'opinione pubblica e la presenza di veto points possono essere sufficienti per generare un certo scetticismo nei confronti di una strategia altamente conflittuale. Potrebbe tuttavia verificarsi la necessità di un processo di ap- 
prendimento politico che passa attraverso tentativi non riusciti di liberalizzazione unilaterale. Si pensi, ad esempio, al ruolo svolto da Berlusconi in Italia.

Ancora più cruciale è forse la convinzione degli attori politici più rilevanti (in particolare, sindacati e partiti di sinistra) dell'impraticabilità della strategia dell'immobilismo. Tali attori potrebbero avere un orizzonte temporale limitato e prestare attenzione agli elementi orientati ad accrescere la sostenibilità della policy (ad esempio, per i sistemi delle pensioni di vecchiaia). Ross (1998) e Levy (1999) hanno rilevato come gli attori si trovino politicamente nelle condizioni di promuovere le riforme come necessarie, volte alla rivitalizzazione del contratto sociale e non al suo smantellamento ${ }^{14}$.

Cosa convince tali attori della necessità della riforma? Mentre un paradigma di policy è sempre «costruito socialmente», è probabile che anche circostanze oggettive svolgano un ruolo centrale. E piuttosto difficile che politiche ben istituzionalizzate e apprezzate, frutto molto spesso di un lungo lavoro politico, vengano dismesse con facilità. Nelle parole di Maurizio Ferrera, forse è necessario che gli attori si convincano che «non vi è alcuno status quo» ${ }^{15}$ - in altre parole, che non è più possibile garantire i vigenti assetti di policy. È probabile che ciò determini un processo di apprendimento sociale (circa gli effetti di politiche alternative) e di apprendimento politico (circa le probabilità di conseguire politiche alternative). L'apprendimento sociale può essere guidato da avvenimenti specifici (quali performances allarmanti di alcuni indicatori chiave) o da crisi finanziarie (come una tempesta valutaria). Anche un processo più graduale di apprendimento, in cui tentativi ed errori portano all'abbandono di strategie alternative, può rivelarsi estremamente significativo (Visser e Hemmerlijck 1997). È possibile che entrambi questi processi siano attualmente in atto.

Il processo di apprendimento politico richiederà probabil-

$14 \mathrm{Ne}$ è un esempio tipico il documento sulla terza via e sul nuovo centro sottoscritto da Schroeder e Blair. Il testo contiene infatti forti richiami alla necessità di provvedere ad una consistente ristrutturazione del sistema di welfare, inclusi il contenimento dei costi e la riforma del mercato del lavoro. Nelle parole di uno stretto collaboratore di Schroeder, «non vi sono altre possibilità... Se non ci avviamo su una strada di ristrutturazione del welfare, si andrà al collasso». Un altro collaboratore del cancelliere ha enfatizzato il fatto che «dobbiamo riuscire gradualmente a convincere le persone che non vi sono alternative». «Financial Times», 9 giugno 1999, pp. 1 e 9.

15 Colloquio personale. 
mente un percorso di fallimenti ed errori nel corso di tentativi esplorativi da parte degli attori in cerca di strategie alternative. Si può ipotizzare che l'apprendimento politico determini un esito da «nuovo centro» laddove i partiti e i sindacati sono moderatamente forti. Attori moderatamente forti detengono risorse che sono sufficientemente significative da poter essere messe sul tavolo dei negoziati, ma non così rilevanti da indurre a credere che gli altri attori (un governo di destra o gli imprenditori) in caso di veto si asterranno dal procedere in solitudine. Tale elemento è risultato evidente, ad esempio, nel caso olandese (Visser e Hemmerjick 1997) e italiano (Ferrera e Gualmini 1999 e 2000), in cui le decisioni di negoziare, adottate da attori di «sinistra» o di «centro-sinistra», hanno contribuito anche a determinare le modalità stesse di negoziazione e sono maturate in un contesto di possibile esclusione (Scharpf 1998). Nei Paesi Bassi $\mathrm{e}$ in Germania, i partiti di centro-destra hanno partecipato appieno ai tentativi di riforma.

Se le predisposizioni favorevoli nei confronti della riforma da parte degli attori chiave sono importanti, altrettanto si può dire tuttavia delle condizioni in cui l'«offerta» di tali iniziative di ristrutturazione è possibile. È necessario che gli attori sociali rilevanti e i partiti politici abbiano il desiderio e la capacità di raggiungere accordi e di rispettarli. In proposito, alcuni noti lavori sul corporativismo, che avevano affrontato problemi analoghi nel campo delle relazioni industriali, possono tornare utili. Due fattori appaiono cruciali: il primo risiede nella qualità dei legami tra gli attori sociali e il processo di policy-making che facilita l'effettiva implementazione degli accordi; il secondo consiste nella capacità espressa da parte delle élites di mantenere tra i propri membri un sostegno sufficiente per iniziative impopolari. Tale aspetto riguarda non soltanto $i$ sindacati, ma anche $i$ partiti politici.

L'elenco delle precondizioni sembrerebbe implicare che le prospettive di una riforma negoziata sono piuttosto scarse. Dopotutto, la conclusione di molti contributi della letteratura sul neocorporativismo evidenziava che il crollo era possibile in condizioni di assenza di livelli relativamente elevati di concentrazione organizzativa. Nel corso degli ultimi anni si è assistito tuttavia a un cospicuo incremento di patti sociali, anche (e soprattutto) in assetti istituzionali che potevano considerarsi terreno poco fertile. Attualmente i soggetti in cerca di soluzioni negoziate possiedono due vantaggi importanti. In primo luogo, in li- 
nea con l'analisi proposta nella figura 1, è probabile che si collochino entro lo spazio politico occupato dall'elettore centrale. Il peculiare posizionamento attribuisce a tali soggetti un potere considerevole, soprattutto se essi sono intenzionati ad accettare una certa contrazione di iscritti in cambio di una più consistente influenza sulla policy in questione.

Il secondo vantaggio risiede nella possibilità di trasformare i vizi in virtù. Nei termini impiegati in questo saggio, i pacchetti di ristrutturazione non devono essere limitati al contenimento dei costi e alla mercificazione. Essi possono anche includere consistenti sforzi di modernizzazione. Come è stato osservato, in molti sistemi la necessità di modernizzazione è evidente, e ciò potrebbe facilitare la legittimazione dell'intervento e fornire contestualmente risorse fiscali considerevoli. Se sostenute da un'ampia coalizione «centrista» (formale o informale), dotata della credibilità necessaria per convincere che l'obiettivo è costituito dalla ristrutturazione e non dallo smantellamento, e che tale scelta non ha alternative praticabili, tali proposte possono costituire una formula politica vincente. Paesi bassi, Germania e Italia rivelano la possibilità, limitata ma reale, di una ristrutturazione negoziata dei sistemi di welfare continentali.

\section{Conclusioni}

I tre mondi di welfare differiscono per alcune caratteristiche cruciali che provocano dinamiche politiche distinte di ristrutturazione istituzionale (tab. 1). Più specificamente, i diversi regimi variano in ragione della portata del sostegno in favore dello stato sociale e delle dimensioni e della natura delle pressioni di adattamento. Nei regimi liberali, le spinte verso un adattamento sono state limitate, ma altrettanto limitato è stato il sostegno nei confronti del welfare state esistente. Nei paesi socialdemocratici, il sostegno a favore dello stato sociale è diffuso, mentre le pressioni verso un adattamento sono circoscritte. Infine, nei regimi conservatori sono consistenti sia il sostegno nei confronti dell'esistente regime di welfare, sia le pressioni verso un adattamento.

Le differenze nell'intensità del sostegno, da un lato, e la dimensione e la natura delle pressioni, dall'altro, possono generare distinte coalizioni politiche, distinti percorsi di riforma e (forse più in linea teorica che nella realtà) distinte dimensioni di 
$\mathrm{TAB}$. 1. La riforma dello stato sociale nei tre regimi di welfare

\begin{tabular}{llll}
\hline & Modello liberale & $\begin{array}{l}\text { Modello socialde- } \\
\text { mocratico }\end{array}$ & $\begin{array}{l}\text { Modello conserva- } \\
\text { tore }\end{array}$ \\
\hline $\begin{array}{l}\text { Sostegno politico } \\
\text { allo Stato sociale }\end{array}$ & Moderato & Elevato & Elevato \\
$\begin{array}{l}\text { Pressioni verso l'a- } \\
\text { dattamento }\end{array}$ & Moderato & Moderato & Elevato \\
$\begin{array}{l}\text { Composizione del- } \\
\text { l'agenda di riforma }\end{array}$ & $\begin{array}{l}\text { - Rimercificazione } \\
\text {-Contenimento dei } \\
\text { costi }\end{array}$ & $\begin{array}{l}\text {-Contenimento dei } \\
\text { costi }\end{array}$ & $\begin{array}{l}\text { - Contenimento dei } \\
\text { costi }\end{array}$ \\
$\begin{array}{l}\text { Linea di conflitto } \\
\text { prevalente }\end{array}$ & $\begin{array}{l}\text { Ridimento (ra- } \\
\text { mento neoliberale } \\
\text { vs. mercificazione } \\
\text { compensatoria }\end{array}$ & $\begin{array}{l}\text { Adatamento (ag- } \\
\text { giornamento) }\end{array}$ \\
$\begin{array}{l}\text { cleavage dominan- } \\
\text { ti. Adattamento ne- } \\
\text { goziato e incre- } \\
\text { mentale }\end{array}$ & $\begin{array}{l}\text { Immobilismo vs. } \\
\text { riforma negoziata }\end{array}$ \\
Variabili rilevanti & $\begin{array}{l}\text { Consistenza delle } \\
\text { pressioni economi- } \\
\text { che; concentrazio- } \\
\text { ne dell'autorità po- } \\
\text { litica }\end{array}$ & $\begin{array}{l}\text { Consistenza delle } \\
\text { pressioni economi- } \\
\text { che }\end{array}$ & $\begin{array}{l}\text { Consistenza delle } \\
\text { pressioni economi- } \\
\text { che; vulneralibilità } \\
\text { della coalizione cen- } \\
\text { trista (possibile «in- } \\
\text { vasione di campo») }\end{array}$ \\
\hline
\end{tabular}

criticità nel processo di ristrutturazione. In breve, non vi ̀̀ un'unica «nuova politica» che accompagna la ristrutturazione; vi sono invece dinamiche politiche distinte che si sviluppano in distinte configurazioni di welfare. Nel mondo liberale, la riforma verte sul contenimento dei costi e sulla ri-mercificazione, ed è presente una chiara divisione tra sostenitori, vicini a posizioni neoliberali, di un profondo ridimensionamento e sostenitori di una soluzione più consensuale e compensativa. In un contesto in cui il sostegno nei confronti delle prestazioni sociali è generalmente limitato e l'arena elettorale/legislativa gioca un ruolo centrale, il controllo del governo da parte di un solo partito e il grado di concentrazione dell'autorità politica nelle istituzioni diventano fattori cruciali. Nel mondo socialdemocratico, la riforma è incentrata sul contenimento dei costi e sull'adattamento del sistema di welfare finalizzato al perseguimento, in modo più efficace rispetto al passato, dei tradizionali obiettivi. Nel complesso, la riforma è negoziata, consensuale e incrementale. Nel mondo conservatore, la riforma si basa sul contenimento dei costi e sull'adattamento del sistema di welfare, e pone una si- 
gnificativa enfasi sull'aggiornamento dei «vecchi» programmi che devono essere adattati a nuove domande sociali emergenti. Non essendo percorribile la strada del ridimensionamento neoliberale, il processo politico si sostanzia in una serie di tentativi volti alla costruzione di una qualche possibile coalizione capace di far passare le riforme. La struttura dell'intermediazione degli interessi e dei sistemi di partito - in particolare, la possibilità che tale struttura consenta ai riformatori di superare timori di possibili «invasioni di campo» - è centrale.

Due osservazioni conclusive. La prima è che un fattore cruciale nella strutturazione della riforma in tutte le configurazioni di welfare risiede nella notevole consistenza delle pressioni economiche: forti squilibri hanno un impatto formidabile sulle dinamiche di riforma. Tali squilibri possono dipendere o meno (e in misura più o meno intensa) dalla «globalizzazione», ma resta tuttavia importante evidenziare che non è solo la politica a decidere in che misura e quando un dato paese deve procedere a significative iniziative di riforma. $\mathrm{Ne}$ consegue che probabilmente il destino dei sistemi di welfare maturi dipende dalla specifica performance economica dei vari paesi.

La seconda osservazione è che mentre le agende di riforma variano in misura considerevole a seconda dei tipi di regime, tutte pongono come obiettivo prioritario il contenimento dei costi. Tale caratteristica comune riflette l'attuale momento storico, caratterizzato da una condizione di austerità permanente. Ciononostante, $\mathrm{i}$ sistemi di welfare non sono in via di smantellamento: gli sforzi volti al perseguimento di adattamenti possono invece dare luogo a innovazioni significative e talvolta anche ad un allargamento della gamma delle prestazioni offerte. Tali aggiustamenti però si sviluppano ovunque in un contesto in cui il controllo della spesa pubblica costituisce una preoccupazione centrale, talvolta predominante. I fondamenti della maggior parte dei sistemi di welfare non sono quindi in pericolo, anche se lo Zeitgeist attuale resta poco favorevole a consistenti tentativi di migliorare le prestazioni destinate ai più deboli $\mathrm{o}$, sulla base del riconoscimento dell'esistenza di nuovi rischi sociali, all'inclusione nel sistema di welfare di nuove prestazioni sociali. 
Riferimenti bibliografici

Alber, J. (1998), Recent Developments in Continental Welfare States: Do Austria, Germany, and the Netherlands Prove to be Birds of a Feather?, Paper presentato al $14^{\circ}$ Congresso Mondiale di Sociologia, Montreal, luglio.

Andersen, J.G. (1997), Beyond Retrenchment: Welfare Policies in Denmark in the 1990s, Paper presentato nell'ambito dell'incontro organizzato dall'European Consortium for Political Research, Bergen, settembre.

Arthur, B. (1994), Path Dependence and Increasing Returns in Economics, Ann Arbour, University of Michigan Press.

Baccaro, L. e R. Locke (1996), Public Sector Reform and Union Participation: The Case of the Italian Pension Reform, Working Paper, n. 3922-96-BPS, Sloan School of Management, Cambridge (Mass.).

Bonoli, G., V. George e P. Taylor-Gooby (in stampa), European Welfare Futures: Towards a Theory of Retrenchment, Cambridge, Polity Press.

Bonoli, G. e B. Palier (1997), Reclaiming Welfare: The Politics of French Social Protection Reform, in M. Rhodes (a cura di), Southern European Welfare States, Portland, Frank Cass.

Brady, D.W. e C. Voldron (1998), Revolving Gridlock: Politics and Policy from Carter to Clinton, Boulder, Westview Press.

Castles, F.G. (1996), Needs-Based Strategies of Social Protection in Australia and New Zealand, in Esping-Anderson (1996a), pp. 88-115.

Castles, F.G. e C. Pierson (1996), A New Convergence? Recent Policy Developments in the United Kingdom, Australia, and New Zealand, in «Policy and Politics», n. 3, pp. 233-245.

Clayton, R. e J. Pontusson (1999), Welfare State Retrenchment Revisited: Entitlement Cuts, Public Sector Restructuring, and Inegalitarian Trends in Advanced Capitalist Societies, in «World Politics», n. 1, pp. 67-98.

Collier, D. e S. Levitsky (1997), Democracy with Adjectives: Conceptual Innovation in Comparative Research, in «World Politics», pp. 431-451.

Ebbinghaus, B. (1999), Any Way Out of 'Exit from Work'? Reversing the Entrenched Pathways of Early Retirement in Europe, USA and Japan, Paper presentato all'interno del progetto Max Planck Institut «Adjustment to Economic Internationalization».

Ebbinghaus, B. e P. Manow (1998), Varieties of Welfare Capitalism, Paper inedito.

Esping-Andersen, G. (1985), Politics against Markets, Princeton, Princeton University Press.

- (1990), The Three Worlds of Welfare Capitalism, Princeton, Princeton University Press. 
- (1996), Welfare States Without Work: The Impasse of Labour Shedding and Familism in Continental Social Policy, in Id. (a cura di), Welfare States in Transition: National Adaptations in Global Economies, Thousand Oaks, Sage Publications, pp. 66-87.

- (1999), Social Foundations of Postindustrial Economies, Oxford, Oxford University Press.

Ferrera, M. (1996), Il modello sud-europeo di welfare state, in «Rivista Italiana di Scienza Politica», n. 1, pp. 67-101.

- (1997), The Uncertain Future of the Italian Welfare State, in «West European Politics», n. 1, pp. 231-241.

Ferrera, M. e E. Gualmini (1999), Salvati dall'Europa. Lavoro e welfare in Italia fra gli anni'70 e gli anni'90, Bologna, Il Mulino.

- (2000), Italy: Rescue From Without?, in F. Scharpf e V. Schmidt (a cura di), Welfare and Work in Open Economies, Oxford, Oxford University Press.

Flora, P. (1989), From Industrial to Postindustrial Welfare State?, Annali dell'Istituto di Scienze Sociali, numero monografico, Institute of Social Science, Tokio.

Garrett, G. (1998), Partisan Politics in the Global Economy, Cambridge, Cambridge University Press.

Giaimo, S. e P. Manow (1999), Welfare State Adaptation or Erosion? The Case of Health Care Reform in Britain, Germany, and the United States, in «Comparative Political Studies», dicembre.

Golden, M., M. Wallerstein e P. Lange (1999), Postwar Trade-Union Organization and Industrial Restructuring in Twelve Countries, in H. Kitschelt, P. Lange, G. Marks e J.D. Stephens (a cura di), Continuity and Change in Contemporary Capitalism, Cambridge, Cambridge University Press, pp. 194-230.

Hacker, J. (1998), The Historical Logic of National Health Insurance: Structure and Sequence in the Development of British, Canadian, and U.S. Medical Policy, in «Studies in American Political Development», n. 1, pp. 57-130.

Hall, P.A. (1999), The Political Economy Europe in an Era of Interdependence, in H. Kitschelt et al. (1999), pp. 135-163.

Hemerijck, A. e P. Manow (1998), The Experience of Negotiated Reform in Germany and the Netherlands, paper presentato nell'ambito del progetto Max Planck Institut «The Adjustment to Economic Internationalization», gennaio.

Hills, J. (1998), Thatcherism, New Labour and the Welfare State, in «Center for the Analysis of Social Exlusion Paper», n. 13, London School of Economics.

Huber, E. e J. Stephens (in stampa), Partisan Governance, Women's Employment and the Social Democratic Service State, in «American Sociological Review».

Iversen, T. (1998), The Choices for Scandinavian Social Democracy in 
Comparative Perspective, in «Oxford Review of Economic Policy», n. 1, pp. 59-75.

Iversen, T. e A. Wren (1998), Equality, Employment and Budgetary Restraint: the Trilemma of the Service Economy, in «World Politics», n. 4, pp. 507-546.

Iversen, T., J. Pontusson e D. Soskice (a cura di) (1998), Unions, Employers and Central Banks, Cambridge, Cambridge University Press.

Judis, J.B. (1995), Abandoned Surgery: Business and the Failure of Health Care Reform, in «The American Prospect», n. 21, pp. 65-73.

Kitschelt, H., P. Lange, G. Marks e J.D. Stephens (a cura di) (1999), Continuity and Change in Contemporary Capitalism, Cambridge, Cambridge University Press.

Krehbiel, K. (1998), Pivotal Politics: A Theory of US Lawmaking, Chicago, The University of Chicago Press.

Kohli, M., M. Rein, A.M. Guillemard e H. van Gunsteren (1991), Time for Retirement: Comparative Studies of Early Exit from the Labor Force, Cambridge, Cambridge University Press.

Korpi, W. (1999), Faces of Inequality: Gender, Class and Inequalities in Different Types of Welfare States, Paper inedito, Swedish Institue for Social Research.

Kuhnle. S. e P. Eitrheim (1999), The Scandinavian Model: Trends and Perspectives, WP/23, European University Institute, Paper presentato nell'ambito dei seminari dell'European Forum.

Levy, J. (1999), Vice into Virtue? Progressive Politics and Welfare Reform in Continental Europe, in «Politics and Society», n. 2, pp. 239-273.

Lindbom, A. (1999), Dismantling the Social Democratic Welfare Model? Has the Swedish Welfare State Lost Its Defining Charateristics?, Paper presentato al Workshop 26, European Consortium for Political Research, Mannheim, marzo.

Manow, P. e E. Seils (1999), 'Adjusting Badly': the German Welfare State, Structural Change and the Economy, Paper presentato per il progetto Max Planck Institut «Adjustment to Economic Internationalization», gennaio.

Myles, J. (1996), When Markets Fail: Social Welfare in Canada and the United States, in Esping-Andersen (1996a), pp. 116-140.

- (1999), The Maturation of Canadas Retirement Income System: Income Levels, Income Inequality and Low Income among the Elder$l y$, Paper inedito.

Myles, J. e P. Pierson (1997), Friedman's Revenge: the Reform of 'Liberal' Welfare States in Canada and the United States, in «Politics and Society», pp. 443-472.

- (in stampa), The Comparative Political Economy of Pension Reform, in P. Pierson (in stampa).

North, D.C. (1990), Institutions, Institutional Change, and Economic Performance, Cambridge, Cambridge University Press. 
O'Conner, J., A. Orloff e S. Shaver (1999), States, Families, Markets, Cambridge, Cambridge University Press.

Pierson, C. (1998), Globalisation and the Changing Governance of Welfare States: Superannuation Reform in Australia, in «Global Society», n. 1, pp. 31-47.

Pierson, P. (1994), Dismantling the Welfare State? Reagan, Thatcher and the Politics of Retrenchment, Cambridge, Cambridge University Press.

- (1996), The New Politics of the Welfare State, in «World Politics», n. 2, pp. 143-179.

- (1997), Increasing Returns, Path Dependence, and the Study of Politics, Eui.

- (1998), Irresistible Forces, Immovable Objects: Postindustrial Welfare States Confront Permanent Austerity, in «Journal of European Public Policy», n. 4, pp. 539-560.

- Pierson, P. (a cura di) (in stampa), The New Politics of the Welfare State, Oxford, Oxford University Press.

Pierson, P. e R.K. Weaver (1993), Imposing Losses in Pensions Policy, in R.K. Weaver e B.A. Rockman (a cura di), Do Institutions Matter? Government Capabilities in the United States and Abroad, Washington, D.C., Brookings Institution Press, pp. 110-150.

Ragin, C. (1987), The Comparative Method: Moving Beyond Qualitative and Quantitative Strategies, Berkeley, University of California Press.

Ross, F. (1998), A Framework for Studying Unpopular Policies: Partisan Possibilities, Institutional Liabilities and the Anti-State Agenda, Paper inedito.

Rothstein, B. (1998), Just Institutions Matter, Cambridge, Cambridge University Press.

Scharpf, F.W. (1986), The Joint-Decision Trap: Lessons from German Federalism and European Integration, in «Public Administration», pp. 239-278.

- (1997), Employment and the Welfare State: A Continental Dilemma, Max Planck Institut Working Paper, n. 7.

Schwartz, H. e M. Rhodes (1999), Internationalization and the Liberal Welfare States: the UK, Australia and New Zealand, Paper presentato per il progetto Max Planck Institut «Adjustment to Economic Internationalization».

Shalev, M. (1998), Limits and Alternatives to Multiple Regression in Macro-Comparative Research, Paper presentato alla II Conferenza «Welfare State», Crossroads, Stoccolma, giugno.

Soskice, D. (1999), Divergent Production Regimes: Coordinated and Uncoordinated Market Economies in the 1980s and 1990s, in Kitschelt et al. (1999), pp. 101-134.

Stephens, J.D. (1996), The Scandinavian Welfare States: Achievements and Prospect, in Esping-Andersen (1996a), pp. 32-65. 
Stephens, J.D., E. Huber e L. Ray (1999), The Welfare State in Hard Times, in Kitschelt et al. (1999), pp. 164-193.

Svallfors, S. (1995), The End of Class Politics? Structural Cleavages and Attitudes to Swedish Welfare Policies, in «Acta Sociologica», pp. 53-74.

- (1997), Worlds of Welfare and Attitudes To Redistribution: A Comparison of Eight Western Nations, in «European Sociological Review», n. 3, pp. 283-304.

Swenson, P. (1992), Union Politics, the Welfare State, and Intraclass Conflict in Sweden and Germany, in M. Golden e J. Pontusson (a cura di), Bargaining for Change: Union Politics in North America and Europe, Ithaca, Cornell University Press, pp. 45-76.

Thelen, K. (in stampa), Why German Employers Cannot Bring Themselves to Dismantle the German Model, in T. Iversen, J. Pontusson e D. Soskice (a cura di), Unions, Employers and Cential Bank: Wage Bargaining and Macro-economic Regimes in an Integrating Europe, Cambridge, Cambridge University Press.

Tsebelis, G. (1995), Decision Making in Political Systems: Veto Players in Presidentialism, Parliamentarism, Multicameralism and Multipartyism, in «British Journal of Political Science», pp. 289-325.

Van Kersbergen, K. (1995), Social Capitalism: A Study of Christian Democracy and the Welfare State, London, Routledge e Kegan Paul.

Visser, J. e A. Hemmerijck (1997), 'A Dutch Miracle': Job Growth, Welfare Reform, and Corporatism in the Nederlands, Amsterdam, Amsterdam University Press.

Wilensky, H., (1981), Leftism, Catholicism, and Democratic Corporatism: the Role of Political Parties in Recent Welfare State Development, in P. Flora e A.J. Heidenheimer (a cura di), The Development of Welfare States in Europe and America, New Brunswick, N.J., Transaction Books, pp. 345-382.

Weaver, R.K. (1986), The Politics of Blame Avoidance, in «Journal of Public Policy», pp. 371-398. 